\title{
Why Gravitational Waves Cannot Exist
}

\author{
J.-F. Pommaret \\ CERMICS, Ecole des Ponts Paris Tech, Paris, France \\ Email: jean-francois.pommaret@wanadoo.fr
}

How to cite this paper: Pommaret, J.-F. (2017) Why Gravitational Waves Cannot Exist. Journal of Modern Physics, 8, 2122-2158.

https://doi.org/10.4236/jmp.2017.813130

Received: July 31, 2017

Accepted: December 5, 2017

Published: December 8, 2017

Copyright (c) 2017 by author and Scientific Research Publishing Inc. This work is licensed under the Creative Commons Attribution International License (CC BY 4.0).

http://creativecommons.org/licenses/by/4.0/

\begin{abstract}
The purpose of this short but difficult paper is to revisit the mathematical foundations of both General Relativity (GR) and Gauge Theory (GT) in the light of a modern approach to nonlinear systems of ordinary or partial differential equations, using new methods from Differential Geometry (D.C. Spencer, 1970), Differential Algebra (J.F. Ritt, 1950 and E. Kolchin, 1973) and Algebraic Analysis (M. Kashiwara, 1970). The main idea is to identify the differential indeterminates of Ritt and Kolchin with the jet coordinates of Spencer, in order to study Differential Duality by using only linear differential operators with coefficients in a differential field $K$. In particular, the linearized second order Einstein operator and the formal adjoint of the Ricci operator are both parametrizing the 4 first order Cauchy stress equations but cannot themselves be parametrized. In the framework of Homological Algebra, this result is not coherent with the vanishing of a certain second extension module and leads to question the proper origin and existence of gravitational waves. As a byproduct, we also prove that gravitation and electromagnetism only depend on the second order jets (called elations by E. Cartan in 1922) of the system of conformal Killing equations because any 1 -form with value in the bundle of elations can be decomposed uniquely into the direct sum $(R, F)$ where $R$ is a section of the Ricci bundle of symmetric covariant 2-tensors and the EM field $F$ is a section of the vector bundle of skew-symmetric 2-tensors. No one of these purely mathematical results could have been obtained by any classical approach. Up to the knowledge of the author, it is also the first time that differential algebra in a modern setting is applied to study the specific algebraic feature of most equations to be found in mathematical physics, particularly in GR.
\end{abstract}

\section{Keywords}

General Relativity, Riemann Tensor, Weyl Tensor, Ricci Tensor, Einstein Equations, Elastic Waves, Gravitational Waves, Lie Groups, Lie Pseudogroups, Differential Galois Theory, Spencer Operator, Janet Sequence, Spencer Sequence, 
Differential Modules, Algebraic Analysis, Homological Algebra, Extension Modules, Split Exact Sequence

\section{Introduction}

The first motivation for studying the methods used in this paper has been a $1000 \$$ challenge proposed in 1970 by J. Wheeler in the physics department of Princeton University while the author of this paper was a student of D.C. Spencer in the closeby mathematics department:

Is it possible to express the generic solutions of Einstein equations in vacuum by means of the derivatives of a certain number of arbitrary functions like the potentials for Maxwell equations?

During the next 25 years and though surprising it may look like, no progress at all has been made towards any solution, either positive or negative. We now explain the way we found the (negative) solution of this challenge in 1995 [1].

Let us consider a manifold $X$ of dimension $n$ with local coordinates $x=\left(x^{i}\right)$ $=\left(x^{1}, \cdots, x^{n}\right)$, tangent bundle $T$, cotangent bundle $T^{*}$, vector bundle $S_{q} T^{*}$ of $q$-symmetric covariant tensors and vector bundle $\wedge^{r} T^{*}$ of $r$-skew-symmetric covariant tensors or $r$-forms. The group of isometries $y=f(x)$ of the nondegenerate metric $\omega$ with $\operatorname{det}(\omega) \neq 0$ on $X$ is defined by the nonlinear first order system in Lie form:

$$
\omega_{k l}(f(x)) \partial_{i} f^{k}(x) \partial_{j} f^{l}(x)=\omega_{i j}(x)
$$

Linearizing at the identity transformation $y=x$, we may introduce the corresponding Killing operator $T \rightarrow S_{2} T^{*}: \xi \rightarrow \mathcal{D} \xi=\mathcal{L}(\xi) \omega=\Omega$, which involves the Lie derivative $\mathcal{L}$ and provides twice the so-called infinitesimal deformation tensor of continuum mechanics when $\omega$ is the Euclidean metric. We may consider the linear first order system of Medolaghi equations.

$$
\Omega_{i j} \equiv(\mathcal{L}(\xi) \omega)_{i j} \equiv \omega_{r j}(x) \partial_{i} \xi^{r}+\omega_{i r}(x) \partial_{j} \xi^{r}+\xi^{r} \partial_{r} \omega_{i j}(x)=0
$$

which is in fact a family of systems only depending on the geometric object $\omega$ and its derivatives. Introducing the Christoffel symbols $\gamma$, we may differentiate once and add the operator $\mathcal{L}(\xi) \gamma=\Gamma \in S_{2} T^{*} \otimes T$ with the well known Levi-Civita isomorphism $j_{1}(\omega)=\left(\omega, \partial_{x} \omega\right) \simeq(\omega, \gamma)$ in order to obtain the linear second order system of Medolaghi equations.

$$
\Gamma_{i j}^{k} \equiv(\mathcal{L}(\xi) \gamma)_{i j}^{k} \equiv \partial_{i j} \xi^{k}+\gamma_{r j}^{k}(x) \partial_{i} \xi^{r}+\gamma_{i r}^{k}(x) \partial_{j} \xi^{r}-\gamma_{i j}^{r}(x) \partial_{r} \xi^{k}+\xi^{r} \partial_{r} \gamma_{i j}^{k}(x)=0
$$

Similarly, introducing the Jacobian determinant $\Delta(x)=\operatorname{det}\left(\partial_{i} f^{k}(x)\right)$, the group of conformal transformations of the metric $\omega$ may be defined by the nonlinear first order system in Lie form:

$$
\hat{\omega}_{k l}(f(x)) \Delta^{-\frac{2}{n}}(x) \partial_{i} f^{k}(x) \partial_{j} f^{l}(x)=\hat{\omega}_{i j}(x)
$$

while introducing the metric density $\hat{\omega}_{i j}=|\operatorname{det}(\omega)|^{-\frac{1}{n}} \omega_{i j} \Rightarrow|\operatorname{det}(\hat{\omega})|=1$ as a 
new geometric object, rather than by eliminating a conformal factor as usual. The conformal Killing operator $\xi \rightarrow \hat{\mathcal{D}} \xi=\mathcal{L}(\xi) \hat{\omega}=\hat{\Omega}$ may be defined by linearization as above and we obtain:

$$
\hat{\Omega}_{i j} \equiv \hat{\omega}_{r j}(x) \partial_{i} \xi^{r}+\hat{\omega}_{i r}(x) \partial_{j} \xi^{r}-\frac{2}{n} \hat{\omega}_{i j}(x) \partial_{r} \xi^{r}+\xi^{r} \partial_{r} \hat{\omega}_{i j}(x)=0
$$

We may introduce the $\operatorname{trace} \operatorname{tr}(\Omega)=\omega^{i j} \Omega_{i j}$ with standard notations and obtain therefore $\operatorname{tr}(\hat{\Omega})=0$ because $\hat{\Omega}_{i j}=|\operatorname{det}(\omega)|^{-\frac{1}{n}}\left(\Omega_{i j}-\frac{1}{n} \omega_{i j} \operatorname{tr}(\Omega)\right)$.

The reader may look at [2] [3] [4] [5] [6] for finding other examples of Lie groups or Lie pseudogroups of transformations along the approach initiated by E. Vessiot in 1903 [7].

In classical elasticity, the stress tensor density $\sigma=\left(\sigma^{i j}=\sigma^{j i}\right)$ existing inside an elastic body is a symmetric 2-tensor density introduced by A. Cauchy in 1822 . Integrating by parts the implicit summation $-\frac{1}{2} \sigma^{i j} \Omega_{i j}$, we obtain the Cauchy operator $\sigma \rightarrow \partial_{r} \sigma^{i r}+\gamma_{r s}^{i} \sigma^{r s}=f^{i}$. When $\omega$ is the euclidean metric, the corresponding Cauchy stress equations can be written as $\partial_{r} \sigma^{i r}=f^{i}$ where the right member describes the local density of forces applied to the body, for example gravitation. With zero second member, we study the possibility to "parametrize" the system of PD equations $\partial_{r} \sigma^{i r}=0$, namely to express its general solution by means of a certain number of arbitrary functions or potentials, called stress functions. Of course, the problem is to know about the number of such functions and the order of the parametrizing operator. For $n=1,2,3$ one may introduce the Euclidean metric $\omega=\left(\omega_{i j}=\omega_{j i}\right)$ while, for $n=4$, one may consider the Minkowski metric. A few definitions used thereafter will be provided later on.

- When $n=2$, the stress equations become $\partial_{1} \sigma^{11}+\partial_{2} \sigma^{12}=0, \partial_{1} \sigma^{21}+\partial_{2} \sigma^{22}$ $=0$. Their second order parametrization $\sigma^{11}=\partial_{22} \phi, \sigma^{12}=\sigma^{21}=-\partial_{12} \phi$, $\sigma^{22}=\partial_{11} \phi$ has been provided by George Biddell Airy (1801-1892) in 1863

[8]. It can be simply recovered in the following manner:

$$
\begin{aligned}
& \partial_{1} \sigma^{11}-\partial_{2}\left(-\sigma^{12}\right)=0 \Rightarrow \exists \varphi, \sigma^{11}=\partial_{2} \varphi, \sigma^{12}=-\partial_{1} \varphi \\
& \partial_{2} \sigma^{22}-\partial_{1}\left(-\sigma^{21}\right)=0 \Rightarrow \exists \psi, \sigma^{22}=\partial_{1} \psi, \sigma^{21}=-\partial_{2} \psi \\
& \sigma^{12}=\sigma^{21} \Rightarrow \partial_{1} \varphi-\partial_{2} \psi=0 \Rightarrow \exists \phi, \varphi=\partial_{2} \phi, \psi=\partial_{1} \phi
\end{aligned}
$$

We get the linear second order system:

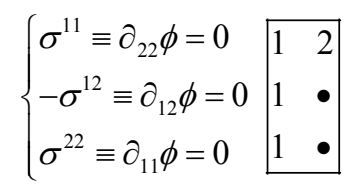

which is involutive with one equation of class 2, 2 equations of class 1 and it is easy to check that the 2 corresponding first order CC are just the stress equations.

When constructing a long prismatic dam with concrete as in [9] [10] or in the 
Introduction of [11], we may transform a problem of 3-dimensional elasticity into a problem of 2-dimensional elasticity by supposing that the axis $x^{3}$ of the dam is perpendicular to the river with $\Omega_{i j}\left(x^{1}, x^{2}\right), \forall i, j=1,2$ and $\Omega_{33}=0$ because of the rocky banks of the river. We may introduce the two Lamé constants $(\lambda, \mu)$ and the Poisson coefficient $v=\lambda / 2(\lambda+\mu)$ in order to describe the usual constitutive relations of an homogeneous isotropic medium as follows, passing from the standard case $n=3$ to the restricted case $n=2$ just by setting:

$$
\begin{aligned}
& \sigma=\frac{1}{2} \lambda \operatorname{tr}(\Omega) \omega+\mu \Omega, \operatorname{tr}(\Omega)=\Omega_{11}+\Omega_{22} \\
& \Rightarrow \mu \Omega=\sigma-\frac{\lambda}{2(\lambda+\mu)} \operatorname{tr}(\sigma) \omega, \operatorname{tr}(\sigma)=\sigma^{11}+\sigma^{22}
\end{aligned}
$$

even though $\sigma^{33}=\frac{1}{2} \lambda\left(\Omega_{11}+\Omega_{22}\right)=\frac{1}{2} \lambda \operatorname{tr}(\Omega) \Rightarrow \sigma^{33}=v\left(\sigma^{11}+\sigma^{22}\right) \neq 0$. Let us consider the right square of the diagram below with locally exact rows:

$$
\begin{array}{rccccc}
2 & \stackrel{\text { Killing }}{\rightarrow} & 3 & \stackrel{\text { Riemann }}{\rightarrow} & 1 & \rightarrow 0 \\
\vdots & & \downarrow \uparrow & & \vdots \\
0 \leftarrow 2 & \stackrel{\text { Cauchy }}{\leftarrow} & 3 & \stackrel{\text { Airy }}{\leftarrow} & 1
\end{array}
$$

Taking into account the formula (5.1.4) of [12] for the linearization of the only component of the Riemann tensor at $\omega$ when $n=2$ and substituting the Airy parametrization, we obtain:

$$
\begin{aligned}
& \operatorname{tr}(R) \equiv d_{11} \Omega_{22}+d_{22} \Omega_{11}-2 d_{12} \Omega_{12}=0 \\
& \Rightarrow \mu \operatorname{tr}(R) \equiv \frac{\lambda+2 \mu}{2(\lambda+\mu)} \Delta \Delta \phi=0 \Rightarrow \Delta \Delta \phi=0
\end{aligned}
$$

where the linearized scalar curvature $\operatorname{tr}(R)$ is allowing to define the Riemann operator in the previous diagram, namely the only compatibility condition (CC) of the Killing operator. It remains to exhibit an arbitrary homogeneous polynomial solution of degree 3 and to determine its 4 coefficients by the boundary pressure conditions on the upstream and downstream walls of the dam. Of course, the Airy potential $\phi$ has nothing to do with the perturbation $\Omega$ of the metric $\omega$ and the Airy parametrization is nothing else but the formal adjoint operator Airy $=a d($ Riemann $)$ of the Riemann operator, linearization of the Riemann tensor over $\omega$, expressing the second order compatibility conditions (CC) of the inhomogeneous system $\mathcal{D} \xi=\Omega$.

- When $n=3$, using now the left square of the following diagram with locally exact rows:

$$
\begin{array}{rcccc}
3 & \stackrel{\text { Killing }}{\rightarrow} & 6 & \multicolumn{2}{c}{\text { Riemann }} \\
\vdots & & \downarrow \uparrow & & 6 \\
0 \leftarrow 3 & \stackrel{ }{\rightarrow} & \vdots \\
0 & 6 & \stackrel{\text { Beltrami }}{\leftarrow} & 6
\end{array}
$$

where the self-adjoint operator $B$ eltrami $=a d($ Riemann $)$ has ben introduced by 
E. Beltrami in 1892. We may substitute the 3-dimensional constitutive relations with Lamé constants $(\lambda, \mu)$ in the Cauchy stress equations and get, when $\boldsymbol{f}=\boldsymbol{g}$ (gravity):

$$
\begin{aligned}
& (\lambda+\mu) \nabla(\nabla \cdot \xi)+\mu \Delta \xi=f \\
& \stackrel{\nabla}{\Rightarrow}(\lambda+2 \mu) \Delta \operatorname{tr}(\Omega)=0 \Rightarrow \Delta \operatorname{tr}(\Omega)=0 \Rightarrow \Delta \operatorname{tr}(\sigma)=0
\end{aligned}
$$

We discover at once that the origin of elastic waves is shifted by one step backwards, from the right square to the left square of the diagram. Indeed, using inertial forces $\boldsymbol{f}=\rho \partial^{2} \boldsymbol{\xi} / \partial t^{2}$ for a medium with mass $\rho$ per unit volume in the right member of Cauchy stress equations because of Newton law and the vector identity $\nabla \wedge(\nabla \wedge \xi)=\nabla(\nabla \cdot \xi)-\Delta \xi$, we discover the existence of two types of elastic waves $\boldsymbol{A} \exp i(\boldsymbol{k} \cdot \boldsymbol{x}-\omega t)$ with wave vector $\boldsymbol{k}$, period $T$, pulsation $\omega=2 \pi / T$ with standard notations, namely the longitudinal and transversal waves with different speeds $v_{T}<v_{L}$, which are really existing because that are responsible for earthquakes [11]:

$$
\left\{\begin{array}{l}
\nabla \cdot \boldsymbol{\xi}=0 \Rightarrow \boldsymbol{k} \cdot \boldsymbol{A}=0 \Rightarrow \mu \Delta \boldsymbol{\xi}=\boldsymbol{f} \Rightarrow v_{T}=\sqrt{\frac{\mu}{\rho}} \\
\nabla \wedge \boldsymbol{\xi}=0 \Rightarrow \boldsymbol{k} \wedge \boldsymbol{A}=0 \Rightarrow(\lambda+2 \mu) \Delta \boldsymbol{\xi}=\boldsymbol{f} \Rightarrow v_{L}=\sqrt{\frac{\lambda+2 \mu}{\rho}}
\end{array}\right.
$$

It is this comment that pushed me to use the formal adjoint of an operator, knowing already that an operator and its (formal) adjoint have the same differential rank (See later on). In the case of the conformal Killing operator, the second order CC are generated by the Weyl operator, linearization of the Weyl tensor over $\hat{\omega}$ when $n \geq 4$. The particular situation pour $n=3$ will be studied in the last section and its corresponding 5 third order CC are not known after one century [6]. Finally, the Bianchi operator describing the CC of the Riemann operator does not appear in this scheme.

Summarizing what we have just said, the study of elastic waves in continuum mechanics only depends on group theory because it has only to do with one differential sequence and its formal adjoint, combined together by means of constitutive relations. We have proved in many books [4] [5] and in [6] [13] [14] that the situation is similar for Maxwell equations, a result leading therefore to revisit the mathematical foundations of both General Relativity (GR) and Gauge Theory (GT), thus also of Electromagnetism (EM).

Knowing already M.P. Malliavin as I gave a seminar on the "Deformation Theory of Algebraic and Geometric Structures" [6] [15], I presented in 1995 a seminar at IHP in Paris, proving the impossibility to parametrize Einstein equations, a result I just found [1]. One of the participants called my attention on a recently published translation from japanese of the 1970 master thesis of $\mathrm{M}$. Kashiwara that he just saw on display in the library of the Institute [16]. This has been the true starting of the story because I discovered that the duality involved in the preceding approach to physics was only a particular example of a much 
more sophisticated framework having to do with homological algebra [11] [17] [18] [19] [20] [21].

Let us explain this point of view by means of an elementary example. With $\partial_{22} \xi=\eta^{2}, \partial_{12} \xi=\eta^{1}$ for $\mathcal{D}$, we get $\partial_{1} \eta^{2}-\partial_{2} \eta^{1}=\zeta$ for the CC $\mathcal{D}_{1}$. Then $\operatorname{ad}\left(\mathcal{D}_{1}\right)$ is defined by $\mu^{2}=-\partial_{1} \lambda, \mu^{1}=\partial_{2} \lambda$ while $\operatorname{ad}(\mathcal{D})$ is defined by $v=\partial_{12} \mu^{1}+\partial_{22} \mu^{2}$ but the CC of $\operatorname{ad}\left(\mathcal{D}_{1}\right)$ are generated by $v^{\prime}=\partial_{1} \mu^{1}+\partial_{2} \mu^{2}$. Using operators, we have the two differential sequences:

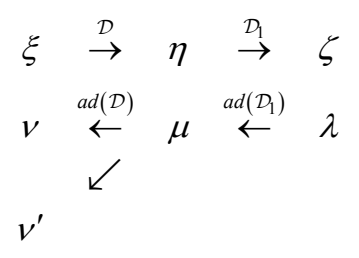

where $\mathcal{D}_{1}$ generates the CC of $\mathcal{D}$ in the upper sequence but $\operatorname{ad}(\mathcal{D})$ does not generate the CC of $\operatorname{ad}\left(\mathcal{D}_{1}\right)$ in the lower sequence, even though $\mathcal{D}_{1} \circ \mathcal{D}=0$ $\Rightarrow \operatorname{ad}(\mathcal{D}) \circ \operatorname{ad}\left(\mathcal{D}_{1}\right)=0$, contrary to what happened in the previous diagram. We shall see that this comment brings the need to introduce the first extension module $\operatorname{ext}^{1}(M)$ of the differential module $M$ determined by $\mathcal{D}$.

In a more intrinsic setting, using the same notation for a vector bundle and its set of (local) sections, we shall have:

$$
\begin{aligned}
& E \stackrel{\mathcal{D}}{\rightarrow} F \\
& \wedge^{n} T^{*} \otimes E^{*} \stackrel{d d(\mathcal{D})}{\leftarrow} \wedge^{n} T^{*} \otimes F^{*}
\end{aligned}
$$

In the meantime, following U. Oberst [22] [23], a few persons were trying to adapt these methods to control theory and, thanks to J.L. Lions, I have been able to advertise about this new approach in a european course, held with succes during 6 years [5] and continued for 5 other years in a slightly different form [24]. By chance I met A. Quadrat, a good PhD student interested by control and computer algebra and we have been staying alone because the specialists of Algebraic Analysis were pure mathematicians, not interested at all by applications. As a byproduct, it is rather strange to discover that the impossibility to parametrize Einstein equations, that we shall prove in Section 4, has never been acknowledged by physicists but can be found in a book on control because it is now known that a control system is controllable if and only if it is parametrizable [24] [25].

The following example of a double pendulum will prove that this result, still not acknowledged today by engineers, is not evident at all. For this, let us consider two pendula of respective length $l_{1}$ and $l_{2}$ attached at the ends of a rigid bar sliding horizontally with a reference position $x(t)$. If the pendula move with a respective (small) angle $\theta_{1}(t)$ and $\theta_{2}(t)$ with respect to the vertical, it is easy to prove from the Newton principle that the equations of the movements does not depend on the respective masses $m_{1}$ and $m_{2}$ of the pendula but only depend on the respective lengths and gravity $g$ along the two formulas: 


$$
d^{2} x+l_{1} d^{2} \theta_{1}+g \theta_{1}=0, \quad d^{2} x+l_{2} d^{2} \theta_{2}+g \theta_{2}=0
$$

where $d=d_{t}$ is the standard time derivative. It is experimentally visible and any reader can check it with a few dollars, that the system is controllable, that is the angles can reach any prescribed (small) values in a finite time when starting from equilibrium, if and only if $l_{1} \neq l_{2}$ and, in this case, we have the following (injective) $4^{\text {th }}$ order parametrization:

$$
\begin{gathered}
-l_{1} l_{2} d^{4} \phi-g\left(l_{1}+l_{2}\right) d^{2} \phi-g^{2} \phi=x, \quad l_{2} d^{4} \phi+g d^{2} \phi=\theta_{1}, \quad l_{1} d^{4} \phi+g d^{2} \phi=\theta_{2} \\
\Rightarrow\left(l_{2}-l_{1}\right) g^{2} \phi=\left(l_{1}-l_{2}\right) x+\left(l_{1}\right)^{2} \theta_{1}-\left(l_{2}\right)^{2} \theta_{2}
\end{gathered}
$$

of course, if $l_{1}=l_{2}=l$, the system cannot be controllable because, setting $\theta=\theta_{1}-\theta_{2}$, we obtain by substraction $l d^{2} \theta+g \theta=0$ and thus $\theta(0)=0$, $d \theta(0)=0 \Rightarrow \theta(t)=0$.

We end this Introduction explaining on a simple example why the second extension module $\operatorname{ext}^{2}(M)$ must also be considered, especially in the study of Einstein equations, though surprising it may look like. To make a comparison, let us consider the following well known Poincaré sequence:

$$
\wedge^{0} T^{*} \stackrel{d}{\rightarrow} \wedge^{1} T^{*} \stackrel{d}{\rightarrow} \wedge^{2} T^{*} \stackrel{d}{\rightarrow} \cdots \stackrel{d}{\rightarrow} \wedge^{n-1} T^{*} \stackrel{d}{\rightarrow} \wedge^{n} T^{*} \rightarrow 0
$$

where $d: \omega=\omega_{I} d x^{I} \rightarrow \partial_{i} \omega_{I} d x^{i} \wedge d x^{I}$ is the exterior derivative. When $n=3$, we have:

$$
\begin{aligned}
& \wedge^{0} T^{*} \stackrel{d}{\rightarrow} \wedge^{1} T^{*} \stackrel{d}{\rightarrow} \wedge^{2} T^{*} \stackrel{d}{\rightarrow} \wedge^{3} T^{*} \rightarrow 0 \Leftrightarrow \phi \stackrel{\text { grad }}{\rightarrow} \xi \stackrel{\text { curl }}{\rightarrow} \eta \stackrel{\text { div }}{\rightarrow} \zeta \rightarrow 0 \\
& 0 \leftarrow \wedge^{3} T^{*} \stackrel{a d(d)}{\leftarrow} \wedge^{2} T^{*} \stackrel{a d(d)}{\leftarrow} \wedge^{1} T^{*} \stackrel{a d(d)}{\leftarrow} \wedge^{0} T^{*} \Leftrightarrow 0 \leftarrow \theta \stackrel{\text { div }}{\leftarrow} \nu \stackrel{\text { curl }}{\leftarrow} \mu \stackrel{\text { grad }}{\leftarrow} \lambda
\end{aligned}
$$

From their definition it follows that div is parametrized by curl while curl is parametrized by grad. Also, in local coordinates, we have ad (div)= - grad, $\operatorname{ad}(\mathrm{curl})=\mathrm{curl}, \mathrm{ad}(\mathrm{grad})=-\mathrm{div}$ and the adjoint sequence is also the Poincaré sequence up to the sign. Let us nevertheless consider the new (minimal) parametrization of div obtained by setting $\xi^{3}=0$, namely [26] [27]:

$$
\begin{aligned}
& d_{2} \xi^{3}-d_{3} \xi^{2}=\eta^{1}, d_{3} \xi^{1}-d_{1} \xi^{3}=\eta^{2}, d_{1} \xi^{2}-d_{2} \xi^{1}=\eta^{3} \\
& \Rightarrow-d_{3} \xi^{2}=\eta^{1}, d_{3} \xi^{1}=\eta^{2}, d_{1} \xi^{2}-d_{2} \xi^{1}=\eta^{3}
\end{aligned}
$$

If we define the differential rank of an operator by the maximum number of differentially independent second member, this is clearly an involutive differential operator with differential rank equal to 2 because $\left(\xi^{1}, \xi^{2}\right)$ can be given arbitrarily and thus $\left(\eta^{1}, \eta^{2}\right)$ can be given arbitrarily or, equivalently, because the differential rank of $d i v$ is of course equal to 1 as $d i v$ has no CC. Now, the involutive system $d_{3} \xi^{2}=0, d_{3} \xi^{1}=0, d_{1} \xi^{2}-d_{2} \xi^{1}=0$ canot be parametrized by one arbitrary function because both $\xi^{1}$ and $\xi^{2}$ are autonomous in the sense that they both satisfy to at least one partial differential equation (PDE). Accordingly, we discover that div can be parametrized by the curl through 3 arbitrary functions $\left(\xi^{1}, \xi^{2}, \xi^{3}\right)$ where $\xi^{3}$ may be given arbitrarily, the curl being itself parametrized by the grad, but div can also be parametrized by another operator with less arbitrary functions or potentials 
which, in turn, cannot be parametrized again. Such a situation is similar to the one met in hunting rifles that may have one, two or more trigger mechanisms that can be used successively. It happens that the possibility to have one parametrization of div is an intrinsic property described by the vanishing of $\operatorname{ext}^{1}(M)$ where the differential module $M$ is determined by grad while the property to have two successive parametrizations is an intrinsic property described by the vanishing of $\operatorname{ext}^{1}(M)$ as we just said plus the vanishing of the second extension module $\operatorname{ext}^{2}(M)$, and so on, but such a result has no classical interpretation. It follows that certain parametrizations are "better" than others and no student should even imagine the minimal parametrization of div that we have presented above. A similar procedure has been adopted by J.C. Maxwell [28] and G. Morera [29] when they modified the parametrization of the Cauchy stress equations obtained by E. Beltrami in 1892 (see [30] and [31] for more details and references or [32] [33] and [34] [35] for computer algebra calculations).

It is clear from the beginning of this Introduction that an isometry is a solution of a nonlinear system in Lie form [2] [5] [6] and that we have linearized this system over the identity transformation in order to study elastic waves. However, in general, no explicit solution may be known but most nonlinear systems of OD or PD equations of mathematical physics (constant riemannian curvature is a good example in [36]) are defined by differential polynomials. This is particularly clear for riemannian, conformal, complex, contact, symplectic or unimodular structures on manifolds [6]. Hence, in Section 2 we shall provide the main results that exist in the formal theory of systems of nonlinear PD equations in order to construct a formal linearization. The proof of many results is quite difficult as it involves delicate chases in 3-dimensional diagrams [2] [5] [11]. In physics, the linear system obtained may have coefficients in a certain differential field and we shall need to revisit differential algebra in Section 3 because Spencer and Kolchin never clearly understood that their respective works could be combined. It will follow that the linear systems will have coefficients in a differential field $K$ and we shall have to introduce the ring $D=K[d]=K\left[d_{1}, \cdots, d_{n}\right]$ of differential operators with coefficients in $K$, which is even an integral domain. This fact will be particularly useful in order to revisit differential duality in Section 4 before applying it to physics in Section 5 and concluding in the last Section 6. This paper is an extended and improved version of a series of lectures given at the Albert Einstein Institute (Berlin/ Postdam), october 23-27, 2017, under the title: "General Relativity and Gauge Theory: Beyond the Mirror".

These purely mathematical results question the origin and existence of gravitational waves.

\section{Differential Geometry}

If $X$ is a manifold with local coordinates $\left(x^{i}\right)$ for $i=1, \cdots, n=\operatorname{dim}(X)$, let $\mathcal{E}$ be a fibered manifold over $X$ with $\operatorname{dim}_{X}(\mathcal{E})=m$, that is a manifold with local 
coordinates $\left(x^{i}, y^{k}\right)$ for $i=1, \cdots, n$ and $k=1, \cdots, m$ simply denoted by $(x, y)$, projection $\pi: \mathcal{E} \rightarrow X:(x, y) \rightarrow(x)$ and changes of local coordinates $\bar{x}=\varphi(x), \bar{y}=\psi(x, y)$. If $\mathcal{E}$ and $\mathcal{F}$ are two fibered manifolds over $X$ with respective local coordinates $(x, y)$ and $(x, z)$, we denote by $\mathcal{E} \times_{X} \mathcal{F}$ the fibered product of $\mathcal{E}$ and $\mathcal{F}$ over $X$ as the new fibered manifold over $X$ with local coordinates $(x, y, z)$. We denote by $f: X \rightarrow \mathcal{E}:(x) \rightarrow(x, y=f(x))$ a global section of $\mathcal{E}$, that is a map such that $\pi \circ f=i d_{X}$ but local sections over an open set $U \subset X$ may also be considered when needed. Under a change of coordinates, a section transforms like $\bar{f}(\varphi(x))=\psi(x, f(x))$ and the derivatives transform like:

$$
\frac{\partial \bar{f}^{l}}{\partial \bar{x}^{r}}(\varphi(x)) \partial_{i} \varphi^{r}(x)=\frac{\partial \psi^{l}}{\partial x^{i}}(x, f(x))+\frac{\partial \psi^{l}}{\partial y^{k}}(x, f(x)) \partial_{i} f^{k}(x)
$$

We may introduce new coordinates $\left(x^{i}, y^{k}, y_{i}^{k}\right)$ transforming like:

$$
\bar{y}_{r}^{l} \partial_{i} \varphi^{r}(x)=\frac{\partial \psi^{l}}{\partial x^{i}}(x, y)+\frac{\partial \psi^{l}}{\partial y^{k}}(x, y) y_{i}^{k}
$$

We shall denote by $J_{q}(\mathcal{E})$ the $q$-jet bundle of $\mathcal{E}$ with local coordinates $\left(x^{i}, y^{k}, y_{i}^{k}, y_{i j}^{k}, \cdots\right)=\left(x, y_{q}\right)$ called jet coordinates and sections $f_{q}:(x) \rightarrow$ $\left(x, f^{k}(x), f_{i}^{k}(x), f_{i j}^{k}(x), \cdots\right)=\left(x, f_{q}(x)\right)$ transforming like the sections $j_{q}(f):(x) \rightarrow\left(x, f^{k}(x), \partial_{i} f^{k}(x), \partial_{i j} f^{k}(x), \cdots\right)=\left(x, j_{q}(f)(x)\right)$ where both $f_{q}$ and $j_{q}(f)$ are over the section $f$ of $\mathcal{E}$. It will be useful to introduce a multi-index $\mu=\left(\mu_{1}, \cdots, \mu_{n}\right)$ with length $|\mu|=\mu_{1}+\cdots+\mu_{n}$ and to set $\mu+1_{i}=\left(\mu_{1}, \cdots, \mu_{i-1}, \mu_{i}+1, \mu_{i+1}, \cdots, \mu_{n}\right)$. Finally, a jet coordinate $y_{\mu}^{k}$ is said to be of class $i$ if $\mu_{1}=\cdots=\mu_{i-1}=0, \mu_{i} \neq 0$. As the background will always be clear enough, we shall use the same notation for a vector bundle or a fibered manifold and their sets of sections [2] [11]. We finally notice that $J_{q}(\mathcal{E})$ is a fibered manifold over $X$ with projection $\pi_{q}$ while $J_{q+r}(\mathcal{E})$ is a fibered manifold over $J_{q}(\mathcal{E})$ with projection $\pi_{q}^{q+r}, \forall r \geq 0$ [2] [3] [4] [5].

DEFINITION 2.1: A (nonlinear) system of order $q$ on $\mathcal{E}$ is a fibered submanifold $\mathcal{R}_{q} \subset J_{q}(\mathcal{E})$ and a global or local solution of $\mathcal{R}_{q}$ is a section $f$ of $\mathcal{E}$ over $X$ or $U \subset X$ such that $j_{q}(f)$ is a section of $\mathcal{R}_{q}$ over $X$ or $U \subset X$.

DEFINITION 2.2: When the changes of coordinates have the linear form $\bar{x}=\varphi(x), \bar{y}=A(x) y$, we say that $\mathcal{E}$ is a vector bundle over $X$. Vector bundles will be denoted by capital letters $C, E, F$ and will have sections denoted by $\xi, \eta, \zeta$. In particular, we shall denote as usual by $T=T(X)$ the tangent bundle of $X$, by $T^{*}=T^{*}(X)$ the cotangent bundle, by $\wedge^{r} T^{*}$ the bundle of $r$-forms and by $S_{q} T^{*}$ the bundle of $q$-symmetric covariant tensors. When the changes of coordinates have the form $\bar{x}=\varphi(x), \bar{y}=A(x) y+B(x)$ we say that $\mathcal{E}$ is an affine bundle over $X$ and we define the associated vector bundle $\mathcal{E}$ over $X$ by the local coordinates $(x, v)$ changing like $\bar{x}=\varphi(x), \bar{v}=A(x) v$.

DEFINITION 2.3: If the tangent bundle $T(\mathcal{E})$ has local coordinates $(x, y, u, v)$ changing like $\bar{u}^{j}=\partial_{i} \varphi^{j}(x) u^{i}, \bar{v}^{l}=\frac{\partial \psi^{l}}{\partial x^{i}}(x, y) u^{i}+\frac{\partial \psi^{l}}{\partial y^{k}}(x, y) v^{k}$, we may introduce the vertical bundle $V(\mathcal{E}) \subset T(\mathcal{E})$ as a vector bundle over $\mathcal{E}$ 
with local coordinates $(x, y, v)$ obtained by setting $u=0$ and changes $\bar{v}^{l}=\frac{\partial \psi^{l}}{\partial y^{k}}(x, y) v^{k}$. Of course, when $\mathcal{E}$ is an affine bundle over $X$ with associated vector bundle $E$ over $X$, we have $V(\mathcal{E})=\mathcal{E} \times{ }_{X} E$. With a slight abuse of language, we shall set $E=V(\mathcal{E})$ as a vector bundle over $\mathcal{E}$.

For a later use, if $\mathcal{E}$ is a fibered manifold over $X$ and $f$ is a section of $\mathcal{E}$, we denote by $f^{-1}(V(\mathcal{E}))$ the reciprocal image of $V(\mathcal{E})$ by $f$ as the vector bundle over $X$ obtained when replacing $(x, y, v)$ by $(x, f(x), v)$ in each chart. A similar construction may also be done for any affine bundle over $\mathcal{E}$. Loking at the transition rules of $J_{q}(\mathcal{E})$, we deduce easily the following results:

PROPOSITION 2.4: $J_{q}(\mathcal{E})$ is an affine bundle over $J_{q-1}(\mathcal{E})$ modeled on $S_{q} T^{*} \otimes_{\mathcal{E}} E$ but we shall not specify the tensor product in general.

PROPOSITION 2.5: There is a canonical isomorphism $V\left(J_{q}(\mathcal{E})\right) \simeq$ $J_{q}(V(\mathcal{E}))=J_{q}(E)$ of vector bundles over $J_{q}(\mathcal{E})$ given by setting $v_{\mu}^{k}=v_{, \mu}^{k}$ at any order and a short exact sequence:

$$
0 \rightarrow S_{q} T^{*} \otimes E \rightarrow J_{q}(E) \stackrel{\pi_{q-1}^{q}}{\rightarrow} J_{q-1}(E) \rightarrow 0
$$

of vector bundles over $J_{q}(\mathcal{E})$ allowing to establish a link with the formal theory of linear systems.

PROPOSITION 2.6: There is an exact sequence:

$$
0 \rightarrow \mathcal{E} \stackrel{j_{q+1}}{\rightarrow} J_{q+1}(\mathcal{E}) \stackrel{D}{\rightarrow} T^{*} \otimes J_{q}(E)
$$

where $D f_{q+1}=j_{1}\left(f_{q}\right)-f_{q+1}$ is over $f_{q}$ with components $\left(D f_{q+1}\right)_{\mu, i}^{k}=\partial_{i} f_{\mu}^{k}$ $-f_{\mu+1_{i}}^{k}$ is called the (nonlinear) Spencer operator. As $J_{q+1}(\mathcal{E}) \subset J_{1}\left(J_{q}(\mathcal{E})\right)$, there is an induced exact sequence:

$$
0 \rightarrow \mathcal{E} \stackrel{j_{q}}{\rightarrow} J_{q}(\mathcal{E}) \stackrel{D_{1}}{\rightarrow} T^{*} \otimes J_{q}(E) / S_{q+1} T^{*} \otimes E
$$

where $D_{1}$ is called the first Spencer operator.

DEFINITION 2.7: If $\mathcal{R}_{q} \subset J_{q}(\mathcal{E})$ is a system of order $q$ on $\mathcal{E}$, then $\mathcal{R}_{q+1}=\rho_{1}\left(\mathcal{R}_{q}\right)=J_{1}\left(\mathcal{R}_{q}\right) \cap J_{q+1}(\mathcal{E}) \subset J_{1}\left(J_{q}(\mathcal{E})\right)$ is called the first prolongation of $\mathcal{R}_{q}$ and we may define the subsets $\mathcal{R}_{q+r}$. In actual practice, if the system is defined by $\operatorname{PDE} \Phi^{\tau}\left(x, y_{q}\right)=0$ the first prolongation is defined by adding the PDE $\quad d_{i} \Phi^{\tau} \equiv \partial_{i} \Phi^{\tau}+y_{\mu+1_{i}}^{k} \partial \Phi^{\tau} / \partial y_{\mu}^{k}=0$. accordingly, $f_{q} \in \mathcal{R}_{q} \Leftrightarrow \Phi^{\tau}\left(x, f_{q}(x)\right)$ $=0$ and $f_{q+1} \in \mathcal{R}_{q+1} \Leftrightarrow \partial_{i} \Phi^{\tau}+f_{\mu+1_{i}}^{k}(x) \partial \Phi^{\tau} / \partial y_{\mu}^{k}=0$ as identities on $X$ or at least over an open subset $U \subset X$. Differentiating the first relation with respect to $x^{i}$ and substracting the second, we finally obtain:

$$
\left(\partial_{i} f_{\mu}^{k}(x)-f_{\mu+1_{i}}^{k}(x)\right) \partial \Phi^{\tau} / \partial y_{\mu}^{k}=0 \Rightarrow D f_{q+1} \in T^{*} \otimes R_{q}
$$

and the Spencer operator restricts to $D: \mathcal{R}_{q+1} \rightarrow T^{*} \otimes R_{q}$. We set $\mathcal{R}_{q+r}^{(1)}=\pi_{q+r}^{q+r+1}\left(\mathcal{R}_{q+r+1}\right)$.

DEFINITION 2.8: The symbol of $\mathcal{R}_{q}$ is the family $g_{q}=R_{q} \cap S_{q} T^{*} \otimes E$ of vector spaces over $\mathcal{R}_{q}$. The symbol $g_{q+r}$ of $\mathcal{R}_{q+r}$ only depends on $g_{q}$ by a direct prolongation procedure. We may define the vector bundle $F_{0}$ over $\mathcal{R}_{q}$ 
by the short exact sequence $0 \rightarrow R_{q} \rightarrow J_{q}(E) \rightarrow F_{0} \rightarrow 0$ and we have the exact induced sequence $0 \rightarrow g_{q} \rightarrow S_{q} T^{*} \otimes E \rightarrow F_{0}$.

Setting $a_{k}^{\tau \mu}\left(x, y_{q}\right)=\partial \Phi^{\tau} / \partial y_{\mu}^{k}\left(x, y_{q}\right)$ whenever $|\mu|=q$ and $\left(x, y_{q}\right) \in \mathcal{R}_{q}$, we obtain:

$$
\begin{aligned}
g_{q}=\left\{v_{\mu}^{k} \in S_{q} T^{*} \otimes E \mid a_{k}^{\tau \mu}\left(x, y_{q}\right) v_{\mu}^{k}=0\right\},|\mu|=q,\left(x, y_{q}\right) \in \mathcal{R}_{q} \\
\Rightarrow g_{q+r}=\rho_{r}\left(g_{q}\right)=\left\{v_{\mu+\nu}^{k} \in S_{q+r} T^{*} \otimes E \mid a_{k}^{\tau \mu}\left(x, y_{q}\right) v_{\mu+\nu}^{k}=0\right\}, \\
\quad|\mu|=q,|v|=r,\left(x, y_{q}\right) \in \mathcal{R}_{q}
\end{aligned}
$$

In general, neither $g_{q}$ nor $g_{q+r}$ are vector bundles over $\mathcal{R}_{q}$.

On $\wedge^{s} T^{*}$ we may introduce the usual bases $\left\{d x^{I}=d x^{i_{1}} \wedge \cdots \wedge d x^{i_{s}}\right\}$ where we have set $I=\left(i_{1}<\cdots<i_{s}\right)$. In a purely algebraic setting, one has:

PROPOSITION 2.9: There exists a map $\delta: \wedge^{s} T^{*} \otimes S_{q+1} T^{*} \otimes E \rightarrow \wedge^{s+1} T^{*}$ $\otimes S_{q} T^{*} \otimes E$ which restricts to $\delta: \wedge^{s} T^{*} \otimes g_{q+1} \rightarrow \wedge^{s+1} T^{*} \otimes g_{q}$ and $\delta^{2}=\delta \circ \delta$ $=0$.

Proof: Let us introduce the family of s-forms $\omega=\left\{\omega_{\mu}^{k}=v_{\mu, I}^{k} d x^{I}\right\}$ and set $(\delta \omega)_{\mu}^{k}=d x^{i} \wedge \omega_{\mu+1_{i}}^{k}$. We obtain at once $\left(\delta^{2} \omega\right)_{\mu}^{k}=d x^{i} \wedge d x^{j} \wedge \omega_{\mu+1_{i}+1_{j}}^{k}=0$ and $a_{k}^{\tau \mu}(\delta \omega)_{\mu}^{k}=d x^{i} \wedge\left(a_{k}^{\tau \mu} \omega_{\mu+1_{i}}^{k}\right)=0$.

Q.E.D.

The kernel of each $\delta$ in the first case is equal to the image of the preceding $\delta$ but this may no longer be true in the restricted case and we set:

DEFINITION 2.10: Let $B_{q+r}^{s}\left(g_{q}\right) \subseteq Z_{q+r}^{s}\left(g_{q}\right)$ and $H_{q+r}^{s}\left(g_{q}\right)=Z_{q+r}^{s}\left(g_{q}\right) / B_{q+r}^{s}\left(g_{q}\right)$ with $H^{s}\left(g_{q}\right)=H_{q}^{s}\left(g_{q}\right)$ be the coboundary space $\operatorname{im}(\delta)$, cocycle space $\operatorname{ker}(\delta)$ and cohomology space at $\wedge^{s} T^{*} \otimes g_{q+r}$ of the restricted $\delta$-sequence which only depend on $g_{q}$ and may not be vector bundles. The symbol $g_{q}$ is said to be s- acyclic if $H_{q+r}^{1}=\cdots=H_{q+r}^{s}=0, \forall r \geq 0$, involutive if it is $\mathrm{n}$-acyclic and finite type if $g_{q+r}=0$ becomes trivially involutive for $\mathrm{r}$ large enough. In particular, if $g_{q}$ is involutive and finite type, then $g_{q}=0$. Finally, $S_{q} T^{*} \otimes E$ is involutive for any $q \geq 0$ if we set $S_{0} T^{*} \otimes E=E$.

Having in mind the example of $x y_{x}-y=0 \Rightarrow x y_{x x}=0$ with rank changing at $x=0$, we have:

PROPOSITION 2.11: If $g_{q}$ is 2-acyclic and $g_{q+1}$ is a vector bundle over $\mathcal{R}_{q}$, then $g_{q+r}$ is a vector bundle over $\mathcal{R}_{q}, \forall r \geq 1$.

Proof: We may define the vector bundle $F_{1}$ over $\mathcal{R}_{q}$ by the following ker/coker exact sequence where we denote by $h_{1} \subseteq T^{*} \otimes F_{0}$ the image of the central map:

$$
0 \rightarrow g_{q+1} \rightarrow S_{q+1} T^{*} \otimes E \rightarrow T^{*} \otimes F_{0} \rightarrow F_{1} \rightarrow 0
$$

and we obtain by induction on $r$ the following commutative and exact diagram of vector bundles over $\mathcal{R}_{q}$ : 


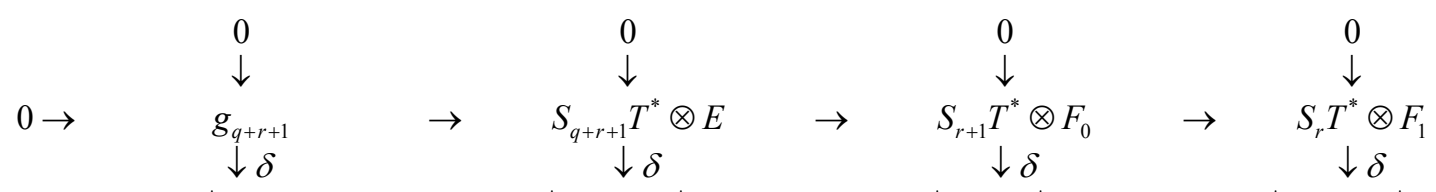

$$
\begin{aligned}
& 0 \rightarrow \quad T^{*} \otimes g_{q+r} \quad \rightarrow \quad T^{*} \otimes S_{q+r} T^{*} \otimes E \quad \rightarrow \quad T^{*} \otimes S_{r} T^{*} \otimes F_{0} \quad \rightarrow \quad T^{*} \otimes S_{r-1} T^{*} \otimes F_{1}
\end{aligned}
$$

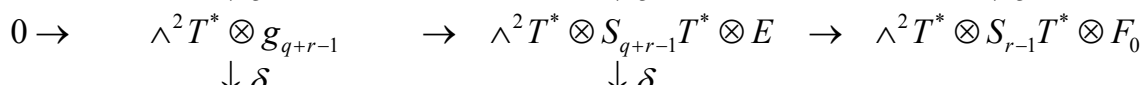

$$
\begin{aligned}
& \wedge^{3} T^{*} \otimes S_{q+r-2} T^{*} \otimes E=\wedge^{3} T^{*} \otimes S_{q+r-2} T^{*} \otimes E
\end{aligned}
$$

where all the maps have been given after Definition 2.9. The image of the central map of the top row is $h_{r+1}=\rho_{r}\left(h_{1}\right)$ and a chase proves that $h_{1}$ is $(s-1)$ -acyclic whenever $g_{q}$ is $s$-acyclic by extending the diagram. The proposition finally follows by upper-semicontinuity from the relation:

$$
\operatorname{dim}\left(g_{q+r+1}\right)+\operatorname{dim}\left(h_{r+1}\right)=\operatorname{mdim}\left(S_{q+r+1} T^{*}\right)
$$

Q.E.D.

LEMMA 2.12: If $g_{q}$ is involutive and $g_{q+1}$ is a vector bundle over $\mathcal{R}_{q}$, then $g_{q}$ is also a vector bundle over $\mathcal{R}_{q}$. In this case, changing linearly the local coordinates if necessary, we may look at the maximum number $\beta$ of equations that can be solved with respect to $v_{n \ldots n}^{k}$ and the intrinsic number $\alpha=m-\beta$ indicates the number of $y$ that can be given arbitrarily.

Using the exactness of the top row in the preceding diagram and a delicate 3-dimensional chase, we have (See [2] and [11], p. 336 for the details):

THEOREM 2.13: If $\mathcal{R}_{q} \subset J_{q}(\mathcal{E})$ is a system of order $q$ on $\mathcal{E}$ such that $g_{q+1}$ is a vector bundle over $\mathcal{R}_{q}$ and $g_{q}$ is 2-acyclic, then there is an exact sequence:

$$
R_{q+r+1} \stackrel{\pi_{q+r}^{q+r+1}}{\rightarrow} R_{q+r} \stackrel{\kappa_{r}}{\rightarrow} S_{r} T^{*} \otimes F_{1}
$$

where $\kappa_{r}$ is called the $r$-curvature and $\kappa=\kappa_{0}$ is simply called the curvature of $\mathcal{R}_{q}$.

We notice that $\mathcal{R}_{q+r+1}=\rho_{r}\left(\mathcal{R}_{q+1}\right)$ and $\mathcal{R}_{q+r}=\rho_{r}\left(\mathcal{R}_{q}\right)$ in the following commutative diagram:

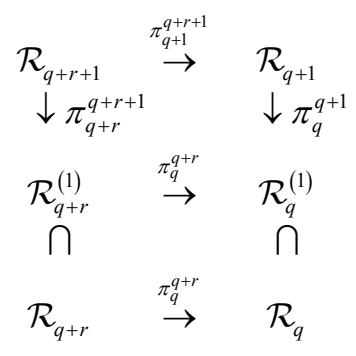

We also have $\mathcal{R}_{q+r}^{(1)} \subseteq \rho_{r}\left(\mathcal{R}_{q}^{(1)}\right)$ because we have successively:

$$
\begin{aligned}
\mathcal{R}_{q+r}^{(1)} & =\pi_{q+r}^{q+r+1}\left(\mathcal{R}_{q+r+1}\right)=\pi_{q+r}^{q+r+1}\left(J_{r}\left(\mathcal{R}_{q+1}\right) \cap J_{q+r+1}(\mathcal{E})\right) \\
& \subseteq J_{r}\left(\pi_{q}^{q+1}\right)\left(J_{r}\left(\mathcal{R}_{q+1}\right)\right) \cap J_{q+r}(\mathcal{E}) \\
& =J_{r}\left(\mathcal{R}_{q}^{(1)}\right) \cap J_{q+r}(\mathcal{E})=\rho_{r}\left(\mathcal{R}_{q}^{(1)}\right)
\end{aligned}
$$

while chasing in the following commutative 3-dimensional diagram: 


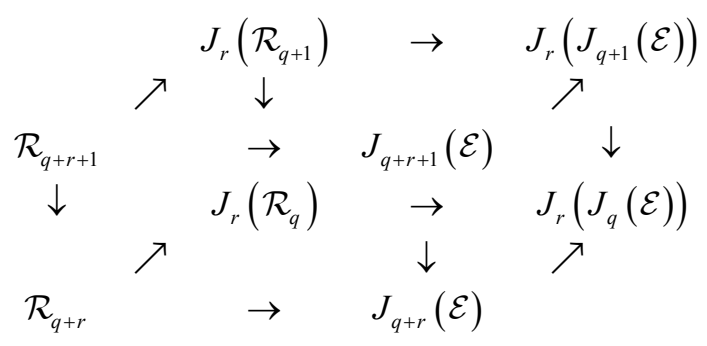

with a well defined map $J_{r}\left(\pi_{q}^{q+1}\right): J_{r}\left(J_{q+1}(\mathcal{E})\right) \rightarrow J_{r}\left(J_{q}(\mathcal{E})\right)$. We finally obtain the following crucial Theorem and its Corollary (Compare to [2], p. 72-74 or [11], p. 340 to [37]):

THEOREM 2.14: Let $\mathcal{R}_{q} \subset J_{q}(\mathcal{E})$ be a system of order $q$ on $\mathcal{E}$ such that $\mathcal{R}_{q+1}$ is a fibered submanifold of $J_{q+1}(\mathcal{E})$. If $g_{q}$ is 2 -acyclic and $g_{q+1}$ is a vector bundle over $\mathcal{R}_{q}$, then we have $\mathcal{R}_{q+r}^{(1)}=\rho_{r}\left(\mathcal{R}_{q}^{(1)}\right)$ for all $r \geq 0$.

DEFINITION 2.15: A system $\mathcal{R}_{q} \subset J_{q}(\mathcal{E})$ is said to be formally integrable if $\pi_{q+r}^{q+r+1}: \mathcal{R}_{q+r+1} \rightarrow \mathcal{R}_{q+r}$ is an epimorphism of fibered manifolds for all $r \geq 1$ and involutive if it is formally integrable with an involutive symbol $g_{q}$. We have the following useful test [2] [37] [38] [39]:

COROLLARY 2.16: Let $\mathcal{R}_{q} \subset J_{q}(\mathcal{E})$ be a system of order $q$ on $\mathcal{E}$ such that $\mathcal{R}_{q+1}$ is a fibered submanifold of $J_{q+1}(\mathcal{E})$. If $g_{q}$ is 2-acyclic (involutive) and if the map $\pi_{q}^{q+1}: \mathcal{R}_{q+1} \rightarrow \mathcal{R}_{q}$ is an epimorphism of fibered manifolds, then $\mathcal{R}_{q}$ is formally integrable (involutive).

This is all what is needed in order to study systems of algebraic ordinary differential (OD) or partial differential (PD) equations.

\section{Differential Algebra}

We now present in an independent manner two OD examples and two PD examples showing the difficulties met when studying differential ideals and ask the reader to revisit them later on while reading the main Theorems. As only a few results will be proved, the interested reader may look at [3] [5] [11] for more details and compare to [40] [41] [42].

EXAMPLE 3.1: If $k=\mathbb{Q}, y$ is a differential indeterminate and $d_{x}$ is a formal derivation, we may set $d_{x} y=y_{x}, d_{x} y_{x}=y_{x x}$ and so on in order to introduce the differential ring $A=k\left[y, y_{x}, y_{x x}, \cdots\right]=k\{y\}$. We consider the (proper) differential ideal $\mathfrak{a} \subset A$ generated by the differential polynomial $P=y_{x}^{2}-4 y$. We have $d_{x} P=2 y_{x}\left(y_{x x}-2\right)$ and a cannot be a prime differential ideal. Hence, looking for the "solutions" of $P=0$, we must have either $y_{x}=0 \Rightarrow y=0$ or $y_{x x}=2$ and thus $y=(x+c)^{2}$ where $c$ should be a "constant" with no clear meaning. However, we have successively:

$$
\begin{aligned}
P \in \mathfrak{a} & \Rightarrow y_{x}\left(y_{x x}-2\right) \in \mathfrak{a} \\
& \Rightarrow y_{x} y_{x x x}+y_{x x}\left(y_{x x}-2\right) \in \mathfrak{a} \\
& \Rightarrow\left(y_{x}\right)^{2} y_{x x x} \in \mathfrak{a} \\
& \Rightarrow y y_{x x x} \in \mathfrak{a} \\
& \Rightarrow y y_{x x x x}+y_{x} y_{x x x} \in \mathfrak{a}
\end{aligned}
$$




$$
\begin{aligned}
& \Rightarrow y_{x}\left(y_{x x x}\right)^{2} \in \mathfrak{a} \\
& \Rightarrow 2 y_{x} y_{x x x} y_{x x x x}+y_{x x}\left(y_{x x x}\right)^{2} \in \mathfrak{a} \Rightarrow 2 y_{x} y_{x x x} y_{x x x x}=-y_{x x}\left(y_{x x x}\right)^{2} \bmod (\mathfrak{a}) \\
& \Rightarrow 4 y_{x x} y_{x x x} y_{x x x x}+2 y_{x}\left(y_{x x x x}\right)^{2}+2 y_{x} y_{x x x} y_{x x x x x}+\left(y_{x x x}\right)^{3} \in \mathfrak{a} \\
& \Rightarrow 4 y_{x x}\left(y_{x x x}\right)^{2} y_{x x x x}+2 y_{x} y_{x x x}\left(y_{x x x x}\right)^{2}+2 y_{x}\left(y_{x x x}\right)^{2} y_{x x x x}+\left(y_{x x x}\right)^{4} \in \mathfrak{a} \\
& \Rightarrow 4 y_{x x}\left(y_{x x x}\right)^{2} y_{x x x x}+2 y_{x} y_{x x x}\left(y_{x x x x}\right)^{2}+\left(y_{x x x}\right)^{4} \in \mathfrak{a} \\
& \Rightarrow 3 y_{x x}\left(y_{x x x}\right)^{2} y_{x x x x}+\left(y_{x x x}\right)^{4} \in \mathfrak{a} \\
& \Rightarrow-6 y_{x} y_{x x x}\left(y_{x x x x}\right)^{2}+\left(y_{x x x}\right)^{4} \in \mathfrak{a} \\
& \Rightarrow\left(y_{x x x}\right)^{5} \in \mathfrak{a} \Rightarrow y_{x x x} \in \operatorname{rad}(\mathfrak{a})
\end{aligned}
$$

and thus $\mathfrak{a}$ is neither prime nor perfect, that is equal to its radical, but $\operatorname{rad}(\mathfrak{a})$ is perfect as it is the intersection of the prime differential ideal generated by $y$ with the prime differential ideal generated by $y_{x}^{2}-4 y$ and $y_{x x}-2$, both containing $y_{x x x}$.

EXAMPLE 3.2: With the notations of the previous Example, let us consider the (proper) differential ideal $\mathfrak{a} \subset A$ generated by the differential polynomial $P=y_{x}^{2}-4 y^{3}$. We have $d_{x} P=2 y_{x}\left(y_{x x}-6 y^{2}\right)$ and $\mathfrak{a}$ cannot be prime differential ideal. Hence, looking for the "solutions" of $P=0$, we must have either $y_{x}=0 \Rightarrow y=0$ or $y_{x}^{2}-4 y^{3}=0$ and $y_{x x}-6 y^{2}=0$. However, we have:

$$
\begin{aligned}
P \in \mathfrak{a} & \Rightarrow y_{x}\left(y_{x x}-6 y^{2}\right) \in \mathfrak{a} \Rightarrow\left(y_{x}\right)^{2}\left(y_{x x}-6 y^{2}\right)^{2} \in \mathfrak{a} \Rightarrow 4 y^{3}\left(y_{x x}-6 y^{2}\right)^{2} \in \mathfrak{a} \\
& \Rightarrow y_{x x}\left(y_{x x}-6 y^{2}\right)+y_{x}\left(y_{x x x}-12 y y_{x}\right) \in \mathfrak{a} \\
& \Rightarrow y_{x x}\left(y_{x x}-6 y^{2}\right)^{2} \in \mathfrak{a} \\
& \Rightarrow\left(y_{x x}\right)^{2}\left(y_{x x}-6 y^{2}\right)^{2}-12 y^{2} y_{x x}\left(y_{x x}-6 y^{2}\right)^{2}+36 y^{4}\left(y_{x x}-6 y^{2}\right)^{2} \in \mathfrak{a} \\
& \Rightarrow\left(y_{x x}-6 y^{2}\right)^{4} \in \mathfrak{a} \Rightarrow y_{x x}-6 y^{2} \in \operatorname{rad}(\mathfrak{a})
\end{aligned}
$$

and thus $\mathfrak{a}$ is neither prime or perfect as before but $\operatorname{rad}(\mathfrak{a})$ is the prime differential ideal generated by $y_{x}^{2}-4 y^{3}$ and $y_{x x}-6 y^{2}$.

EXAMPLE 3.3: If $k=\mathbb{Q}$ as before, $y$ is a differential indeterminate and $\left(d_{1}, d_{2}\right)$ are two formal derivations, let us consider the differential ideal generated by $P_{1}=y_{22}-\frac{1}{2}\left(y_{11}\right)^{2}$ and $P_{2}=y_{12}-y_{11}$ in $k\{y\}$. Using crossed derivatives, we get successively:

$$
\begin{aligned}
P_{1}, P_{2} \in \mathfrak{a} & \Rightarrow y_{112}-y_{111} \in \mathfrak{a}, y_{122}-y_{11} y_{111} \in \mathfrak{a}, y_{222}-y_{11} y_{111} \in \mathfrak{a} \\
& \Rightarrow Q=d_{2} P_{2}-d_{1} P_{1}+d_{1} P_{2}=\left(y_{11}-1\right) y_{111} \in \mathfrak{a} \\
& \Rightarrow d_{1} Q=\left(y_{111}\right)^{2}+\left(y_{11}-1\right) y_{1111} \in \mathfrak{a} \\
& \Rightarrow\left(y_{111}\right)^{3} \in \mathfrak{a} \Rightarrow y_{111} \in \operatorname{rad}(\mathfrak{a})
\end{aligned}
$$

and thus $\mathfrak{a}$ is neither prime nor perfect but $\operatorname{rad}(\mathfrak{a})$ is a perfect differential ideal and even a prime differential ideal $\mathfrak{p}$ because we obtain easily from the last section that the resisual differential ring $k\{y\} / \mathfrak{p} \simeq k\left[y, y_{1}, y_{2}, y_{11}\right]$ is a differential integral domain. Its quotient field is thus the differential field $K=Q(k\{y\} / \mathfrak{p}) \simeq k\left(y, y_{1}, y_{2}, y_{11}\right)$ with the rules:

$$
d_{1} y=y_{1}, d_{1} y_{1}=y_{11}, d_{1} y_{11}=0, d_{2} y=y_{2}, d_{2} y_{1}=y_{11}, d_{2} y_{11}=0
$$


as a way to avoid looking for solutions. The formal linearization is the linear system $\mathcal{R}_{2} \subset J_{2}(E)$ obtained in the last section where it was defined over $\mathcal{R}_{2}$, but not over $K$, by the two linear second order PDE:

$$
Y_{22}-y_{11} Y_{11}=0, \quad Y_{12}-Y_{11}=0
$$

changing slightly the notations for using the letter $v$ only when looking at the symbols. It is at this point that the problem starts because $\mathcal{R}_{2}$ is indeed a fibered manifold with arbitrary parametric jets $\left(y, y_{1}, y_{2}, y_{11}\right)$ but $\mathcal{R}_{3}=\rho_{1}\left(\mathcal{R}_{2}\right)$ is no longer a fibered manifold because the dimension of its symbol changes when $y_{11}=1$. We understand therefore that there should be a close link existing between formal integrability and the search for prime differential ideals or differential fields. The solution of this problem has been provided as early as in 1983 for studying the "Differential Galois Theory" but has never been acknowledged and is thus not known today ([3] [5]). The idea is to add the third order PDE $y_{111}=0$ and thus the linearized PDE $Y_{111}=0$ obtaining therefore a third order involutive system well defined over $K$ with symbol $g_{3}=0$. We invite the reader to treat similarly the two previous examples and to compare.

EXAMPLE 3.4: If $k=\mathbb{Q}$ as before, $y$ is a differential indeterminate and $\left(d_{1}, d_{2}\right)$ are two formal derivations, let us consider the differential ideal generated by $P_{1}=y_{22}-\frac{1}{3}\left(y_{11}\right)^{3}$ and $P_{2}=y_{12}-\frac{1}{2}\left(y_{11}\right)^{2}$ in $k\{y\}$. Using crossed derivatives, we get successively:

$$
\begin{gathered}
P_{1}, P_{2} \in \mathfrak{a} \Rightarrow d_{2} P_{2}-d_{1} P_{1}+y_{11} d_{1} P_{2}=0 \Rightarrow \mathcal{R}_{2} \quad \text { involutive } \\
\Rightarrow y_{222}-\left(y_{11}\right)^{3} y_{111}=0, y_{122}-\left(y_{11}\right)^{2} y_{111}=0, y_{112}-y_{11} y_{111}=0, \cdots
\end{gathered}
$$

and thus $\operatorname{dim}\left(g_{q}\right)=1, \forall q \geq 1$. As the symbol $g_{2}$ is involutive, there is an infinite number of parametric jets $\left(y, y_{1}, y_{2}, y_{11}, y_{111}, \cdots\right)$ and thus $k\{y\} / \mathfrak{a} \simeq$ $k\left[y, y_{1}, y_{2}, y_{11}, y_{111}, \cdots\right]$ is a differential integral domain with $d_{2} y_{2}=y_{22}$ $=\frac{1}{3}\left(y_{11}\right)^{3}, \quad d_{2} y_{11}=y_{112}=y_{11} y_{111}, \ldots$. It follows that $\mathfrak{a}=\mathfrak{p}$ is a prime differential ideal with $\operatorname{rad}(\mathfrak{p})=\mathfrak{p}$. The second order linearized system is:

$$
Y_{22}-\left(y_{11}\right)^{2} Y_{11}=0, \quad Y_{12}-y_{11} Y_{11}=0
$$

is now well defined over the differential field $K=Q(k\{y\} / \mathfrak{p})$ and is involutive.

DEFINITION 3.5: A differential ring is a ring $A$ with a finite number of commuting derivations $\left(\partial_{1}, \cdots, \partial_{n}\right)$ such that $\partial_{i}(a+b)=\partial_{i} a+\partial_{i} b, \partial_{i}(a b)=$ $\left(\partial_{i} a\right) b+a \partial_{i} b$ that can be extended to derivations of the ring of quotients $Q(A)$ by setting $\partial_{i}(a / s)=\left(s \partial_{i} a-a \partial_{i} s\right) / s^{2}, \forall 0 \neq s, a \in A$. We shall suppose from now on that $A$ is even an integral domain and use the differential field $K=Q(A)$. For example, if $x^{1}, \cdots, x^{n}$ are indeterminates over $\mathbb{Q}$, then $\mathbb{Q}[x]=\mathbb{Q}\left[x^{1}, \cdots, x^{n}\right]$ is a differential ring for the standard $\left(\partial_{1}, \cdots, \partial_{n}\right)$ with quotient field $\mathbb{Q}(x)$.

If $K$ is a differential field as above and $\left(y^{1}, \cdots, y^{m}\right)$ are indeterminates over $K$, we transform the polynomial ring $K\{y\}=\lim _{q \rightarrow \infty} K\left[y_{q}\right]$ into a differential 
ring by introducing as usual the formal derivations $d_{i}=\partial_{i}+y_{\mu+1_{i}}^{k} \partial / \partial y_{\mu}^{k}$ and we shall set $K\langle y\rangle=Q(K\{y\})$.

DEFINITION 3.6: We say that $\mathfrak{a} \subset K\{y\}$ is a differential ideal if it is stable by the $d_{i}$, that is if $d_{i} a \in \mathfrak{a}, \forall a \in \mathfrak{a}, \forall i=1, \cdots, n$. We shall also introduce the $\operatorname{radical} \operatorname{rad}(\mathfrak{a})=\left\{a \in A \mid \exists r, a^{r} \in \mathfrak{a}\right\} \supseteq \mathfrak{a}$ and say that $\mathfrak{a}$ is a perfect (or radical) differential ideal if $\operatorname{rad}(\mathfrak{a})=\mathfrak{a}$. If $S$ is any subset of $A$, we shall denote by $\{S\}$ the differential ideal generated by $S$ and introduce the (non-differential) ideal $\rho_{r}(S)=\left\{d_{v} a|a \in S, 0 \leq| v \mid \leq r\right\}$ in $A$.

LEMMA 3.7: If $\mathfrak{a} \subset A$ is a differential ideal, then $\operatorname{rad}(\mathfrak{a})$ is a differential ideal containing $\mathfrak{a}$.

Proof. If $d$ is one of the derivations, we have $a^{r-1} d a=\frac{1}{r} d a^{r} \in\left\{a^{r}\right\}$ and thus:

$$
(r-1) a^{r-2}(d a)^{2}+a^{r-1} d^{2} a \in\left\{a^{r}\right\} \Rightarrow a^{r-2}(d a)^{3} \in\left\{a^{r}\right\} \cdots \Rightarrow(d a)^{2 r-1} \in\left\{a^{r}\right\}
$$

Q.E.D.

LEMMA 3.8: If $\mathfrak{a} \subset K\{y\}$, we set $\mathfrak{a}_{q}=\mathfrak{a} \cap K\left[y_{q}\right]$ with $\mathfrak{a}_{0}=\mathfrak{a} \cap K[y]$ and $\mathfrak{a}_{\infty}=\mathfrak{a}$. We have in general $\rho_{r}\left(\mathfrak{a}_{q}\right) \subseteq \mathfrak{a}_{q+r}$ and the problem will be to know when we may have equality.

We shall say that a differential extension $L=Q(K\{y\} / \mathfrak{p})$ is a finitely generated differential extension of $K$ and we may define the evaluation epimorphism $K\{y\} \rightarrow K\{\eta\} \subset L$ with kernel $\mathfrak{p}$ by calling $\eta$ or $\bar{y}$ the residue of $y$ modulo $p$. If we study such a differential extension $L / K$, by analogy with Section 2, we shall say that $R_{q}$ or $g_{q}$ is a vector bundle over $\mathcal{R}_{q}$ if one can find a certain number of maximum rank determinant $D_{\alpha}$ that cannot be all zero at a generic solution of $\mathfrak{p}_{q}$ defined by differential polynomials $P_{\tau}$, that is to say, according to the Hilbert Theorem of Zeros, we may find polynomials $A_{\alpha}, B_{\tau} \in K\left\{y_{q}\right\}$ such that:

$$
\sum_{\alpha} A_{\alpha} D_{\alpha}+\sum_{\tau} B_{\tau} P_{\tau}=1
$$

The following Lemma will be used in the next important Theorem:

LEMMA 3.9: If $\mathfrak{p}$ is a prime differential ideal of $K\{y\}$, then, for $q$ sufficiently large, there is a polynomial $D \in K\left[y_{q}\right]$ such that $D \notin \mathfrak{p}_{q}$ and:

$$
D \mathfrak{p}_{q+r} \subset \operatorname{rad}\left(\rho_{r}\left(\mathfrak{p}_{q}\right)\right) \subset \mathfrak{p}_{q+r}, \quad \forall r \geq 0
$$

THEOREM 3.10: (Primality test) Let $\mathfrak{p}_{q} \subset K\left[y_{q}\right]$ and $\mathfrak{p}_{q+1} \subset K\left[y_{q+1}\right]$ be prime ideals such that $\mathfrak{p}_{q+1}=\rho_{1}\left(\mathfrak{p}_{q}\right)$ and $\mathfrak{p}_{q+1} \cap K\left[y_{q}\right]=\mathfrak{p}_{q}$. If the symbol $g_{q}$ of the algebraic variety $\mathcal{R}_{q}$ defined by $\mathfrak{p}_{q}$ is 2 -acyclic and if its first prolongation $g_{q+1}$ is a vector bundle over $\mathcal{R}_{q}$, then $\mathfrak{p}=\rho_{\infty}\left(\mathfrak{p}_{q}\right)$ is a prime differential ideal with $\mathfrak{p} \cap K\left[y_{q+r}\right]=\rho_{r}\left(\mathfrak{p}_{q}\right), \forall r \geq 0$.

COROLLARY 3.11: Every perfect differential ideal of $\{y\}$ can be expressed in a unique way as the non-redundant intersection of a finite number of prime differential ideals.

COROLLARY 3.12: (Differential basis) If $\mathfrak{r}$ is a perfect differential ideal of $K\{y\}$, then we have $\mathfrak{r}=\operatorname{rad}\left(\rho_{\infty}\left(\mathfrak{r}_{q}\right)\right)$ for $q$ sufficiently large. 
EXAMPLE 3.13: As $K\{y\}$ is a polynomial ring with an infinite number of variables it is not noetherian and an ideal may not have a finite basis. With $K=\mathbb{Q}, n=1$ and $d=d_{x}$, then $\mathfrak{a}=\left\{y y_{x}, y_{x} y_{x x}, y_{x x} y_{x x x}, \cdots\right\} \Rightarrow\left(y_{x}\right)^{2}+y y_{x x}$ $\in \mathfrak{a} \Rightarrow \operatorname{rad}(\mathfrak{a})=\left\{y_{x}\right\}$ is a prime differential ideal.

PROPOSITION 3.14: If $\zeta$ is differentially algebraic over $K\langle\eta\rangle$ and $\eta$ is differentially algebraic over $K$, then $\zeta$ is differentially algebraic over $K$. Setting $\xi=\zeta-\eta$, it follows that, if $L / K$ is a differential extension and $\xi, \eta \in L$ are both differentially algebraic over $K$, then $\xi+\eta, \xi \eta$ and $d_{i} \xi$ are differentially algebraic over $K$.

If $L=Q(K\{y\} / \mathfrak{p}), \quad M=Q(K\{z\} / \mathfrak{q})$ and $N=Q(K\{y, z\} / \mathfrak{r})$ are such that $\mathfrak{p}=\mathfrak{r} \cap K\{y\}$ and $\mathfrak{q}=\mathfrak{r} \cap K\{z\}$, we have the two towers $K \subset L \subset N$ and $K \subset M \subset N$ of differential extensions and we may therefore define the new tower $K \subseteq L \bigcap M \subseteq\langle L, M\rangle \subseteq N$. However, if only $L / K$ and $M / K$ are known and we look for such an $N$ containing both $L$ and $M$, we may use the universal property of tensor products an deduce the existence of a differential morphism $L \otimes_{K} M \rightarrow N$ by setting $d(a \otimes b)=\left(d_{L} a\right) \otimes b+a \otimes\left(d_{M} b\right)$ whenever $d_{L}\left|K=d_{M}\right| K=\partial$. The construction of an abstract composite differential field amounts therefore to look for a prime differential ideal in $L \otimes_{K} M$ which is a direct sum of integral domains [3].

DEFINITION 3.15: A differential extension $L$ of a differential field $K$ is said to be differentially algebraic over $K$ if every element of $L$ is differentially algebraic over $K$. The set of such elements is an intermediate differential field $K^{\prime} \subseteq L$, called the differential algebraic closure of $K$ in $L$. If $L / K$ is a differential extension, one can always find a maximal subset $S$ of elements of $L$ that are differentially transcendental over $K$ and such that $L$ is differentially algebraic over $K\langle S\rangle$. Such a set is called a differential transcedence basis and the number of elements of $S$ is called the differential transcendence degree of $L / K$.

THEOREM 3.16: The number of elements in a differential basis of $L / K$ does not depent on the generators of $L / K$ and his value is $\operatorname{difftrd}(L / K)=\alpha$. Moreover, if $K \subset L \subset M$ are differential fields, then $\operatorname{difftrd}(M / K)=\operatorname{difftrd}(M / L)+\operatorname{difftrd}(L / K)$.

THEOREM 3.17: If $L / K$ is a finitely generated differential extension, then any intermediate differential field $K^{\prime}$ between $K$ and $L$ is also finitely generated over $K$.

EXAMPLE 3.18: With $k=\mathbb{Q}$, let us introduce the manifolds $X$ with local coordinate $x$ and $Y$ with local coordinates $\left(y^{1}, y^{2}\right)$. We may consider the algebraic Lie pseudogroup $\Gamma \subset$ aut $(Y)$ of (local, invertible) transformations of $Y$ preserving the 1 -form $y^{2} d y^{1}$, that is to say made up by transformations $\bar{y}=g(y)$ solutions of the Pfaffian system $\bar{y}^{2} d \bar{y}^{1}=y^{2} d y^{1}$. Equivalently, we have to look for the invertible solutions of the algebraic first order involutive system $\mathcal{R}_{1} \subset J_{1}(Y \times Y)$ defined over $k\left(y^{1}, y^{2}\right)$ by the first order involutive system of algebraic PD equations in Lie form: 


$$
\bar{y}^{2} \frac{\partial \bar{y}^{1}}{\partial y^{1}}=y^{2}, \bar{y}^{2} \frac{\partial \bar{y}^{1}}{\partial y^{2}}=0 \Rightarrow \frac{\partial\left(\bar{y}^{1}, \bar{y}^{2}\right)}{\partial\left(y^{1}, y^{2}\right)}=1
$$

By chance one can obtain the generic solution $\bar{y}^{1}=g\left(y^{1}\right)$, $\bar{y}^{2}=y^{2} /\left(\partial g\left(y^{1}\right) / \partial y^{1}\right)$ where $g$ is an arbitrary function of one variable. Now, if we introduce a function $y=f(x)$ and consider the corresponding transformations of the jets $\left(y^{1}, y^{2}, y_{x}^{1}, y_{x}^{2}, \cdots\right)$, we obtain the only generating differential invariant $\Phi \equiv \bar{y}^{2} \bar{y}_{x}^{1}=y^{2} y_{x}^{1}$. Hence, setting $K=k\left\langle y^{2} y_{x}^{1}\right\rangle$ and $L=k\left\langle y^{1}, y^{2}\right\rangle$, we have the tower of differential extensions $k \subset K \subset L$. As any intermediate differential field $K \subset K^{\prime} \subset L$ is finitely generated, let us consider $K^{\prime}=k\left\langle y^{2} y_{x}^{1}, y_{x}^{2}\right\rangle$. Then:

$$
\bar{y}_{x}^{2} \frac{\partial \bar{y}^{2}}{\partial y^{1}} y_{x}^{1}+\frac{\partial \bar{y}^{2}}{\partial y^{2}} \Rightarrow \frac{\partial \bar{y}^{2}}{\partial y^{1}}=0, \frac{\partial \bar{y}^{2}}{\partial y^{2}}=1 \Rightarrow \bar{y}^{1}=y^{1}+c s t, \bar{y}^{2}=y^{2}
$$

allows to define a Lie subpseudogroup $\Gamma^{\prime} \subset \Gamma$ with generating differential invariants $y_{x}^{1}, y^{2}$ in such a way that, if we set $K^{\prime \prime}=k\left\langle y_{x}^{1}, y^{2}\right\rangle$, we have the strict inclusions $K \subset K^{\prime} \subset K^{\prime \prime}$ and it does not seem possible to obtain a differential Galois correspondence between algebraic subpseudogroups and intermediate differential fields, similar to the classical one. We have explained in [3] how to overcome this problem but this is out of the scope of this paper. It is finally important to notice that the fundamental differential isomorphism [3] [43] [44]:

$$
Q\left(L \otimes_{K} L\right) \simeq Q\left(L \otimes_{k(y)} k[\Gamma]\right)
$$

is the Hopf dual of the projective limit of the action graph isomorphisms between fibered manifolds:

$$
\mathcal{A}_{q} \times_{X} \mathcal{A}_{q} \simeq \mathcal{A}_{q} \times_{Y} \mathcal{R}_{q}
$$

of fibered dimension $2(q+2)$. The corresponding automorphic system $y^{2} y_{x}^{1}=\omega$ in Lie form where $\omega$ is a geometric object as in the Introduction and its prolongations has been introduced as early as in 1903 by E. Vessiot [7] [45] as a way to study principal homogeneous spaces (PHS) for Lie pseudogroups, namely if $y=f(x)$ is a solution and $\bar{y}=\bar{f}(x)$ is another solution, then there exists one and only one transformation $\bar{y}=g(y)$ of $\Gamma$ such that $\bar{f}=g \circ f$.

This is all what is needed in order to study systems of infinitesimal Lie equations defined, like the classical and conformal Killing systems, over $\mathbb{Q}\langle\omega\rangle$ where $\omega$ is a geometric object solution of a system of algebraic Vessiot structure equations (constant Riemann curvature, zero Weyl tensor).

\section{Differential Duality}

Let $A$ be a unitary ring, that is $1, a, b \in A \Rightarrow a+b, a b \in A, 1 a=a 1=a$ and even an integral domain ( $a b=0 \Rightarrow a=0$ or $b=0$ ) with field of fractions $K=Q(A)$. However, we shall not always assume that $A$ is commutative, that is $a b$ may be different from $b a$ in general for $a, b \in A$. We say that $M={ }_{A} M$ is a left module over $A$ if $x, y \in M \Rightarrow a x, x+y \in M, \forall a \in A$ or a right module $M_{B}$ 
over $B$ if the operation of $B$ on $M$ is $(x, b) \rightarrow x b, \forall b \in B$. If $M$ is a left module over $A$ and a right module over $B$ with $(a x) b=a(x b), \forall a \in A, \forall b \in B, \forall x \in M$, then we shall say that $M={ }_{A} M_{B}$ is a bimodule. Of course, $A={ }_{A} A_{A}$ is a bimodule over itself. We define the torsion submodule $t(M)=\{x \in M \mid \exists 0 \neq a \in A, a x=0\} \subseteq M$ and $M$ is a torsion module if $t(M)=M$ or a torsion-free module if $t(M)=0$. We denote by $h_{A}(M, N)$ the set of morphisms $f: M \rightarrow N$ such that $f(a x)=a f(x)$. We finally recall that a sequence of modules and maps is exact if the kernel of any map is equal to the image of the map preceding it.

When $A$ is commutative, $\operatorname{hom}(M, N)$ is again an $A$-module for the law $(b f)(x)=f(b x) \quad$ as $\quad$ we have $\quad(b f)(a x)=f(b a x)=f(a b x)=a f(b x)=$ $a(b f)(x)$. In the non-commutative case, things are more complicate and, given ${ }_{A} M$ and ${ }_{A} N_{B}$, then $\operatorname{hom}_{A}(M, N)$ becomes a right module over $B$ for the law $(f b)(x)=f(x) b$.

DEFINITION 4.1: A module $F$ is said to be free if it is isomorphic to a (finite) power of $A$ called the rank of $F$ over $A$ and denoted by $r k_{A}(F)$ while the rank $r k_{A}(M)$ of a module $M$ is the rank of a maximum free submodule $F \subset M$. It follows from this definition that $M / F$ is a torsion module. In the sequel we shall only consider finitely presented modules, namely finitely generated modules defined by exact sequences of the type $F_{1} \stackrel{d_{1}}{\rightarrow} F_{0} \stackrel{p}{\rightarrow} M \rightarrow 0$ where $F_{0}$ and $F_{1}$ are free modules of finite ranks $m_{0}$ and $m_{1}$ often denoted by $m$ and $p$ in examples. A module $P$ is called projective if there exists a free module $F$ and another (projective) module $Q$ such that $P \oplus Q \simeq F$.

PROPOSITION 4.2: For any short exact sequence $0 \rightarrow M^{\prime} \stackrel{f}{\rightarrow} M \stackrel{g}{\rightarrow} M^{\prime \prime} \rightarrow 0$, we have the relation $r k_{A}(M)=r k_{A}\left(M^{\prime}\right)+r k_{A}\left(M^{\prime \prime}\right)$, even in the non-commutative case. As a byproduct, if $M$ admits a finite length free resolution $\cdots \stackrel{d_{2}}{\rightarrow} F_{1} \stackrel{d_{1}}{\rightarrow} F_{0}$ $\stackrel{p}{\rightarrow} M \rightarrow 0$, we may introduce the Euler-Poincaré characteristic $\chi_{A}(M)=$ $\sum_{r}(-1)^{r} r k_{A}\left(F_{r}\right)=r k_{A}(M)$ (See [11], p. 469).

The following proposition will be used many times in Section 5, in particular for exhibiting the Weyl tensor from the Riemann tensor ([17], p. 73) ([21], p. 33):

PROPOSITION 4.3: We shall say that the following short exact sequence splits if one of the following equivalent three conditions holds:

$$
0 \rightarrow M^{\prime} \stackrel{\stackrel{u}{\leftarrow}}{\rightarrow} \stackrel{\stackrel{v}{\rightarrow}}{\stackrel{\leftarrow}{\rightarrow}} M^{\prime \prime} \rightarrow 0
$$

- There exists a monomorphism $v: M^{\prime \prime} \rightarrow M$ called lift of $g$ and such that $g \circ v=i d_{M^{\prime \prime}}$.

- There exists an epimorphism $u: M \rightarrow M^{\prime}$ called lift of $f$ and such that $u \circ f=i d_{M^{\prime}}$.

- There exist isomorphisms $\varphi=(u, g): M \rightarrow M^{\prime} \oplus M^{\prime \prime}$ and $\psi=f+v: M^{\prime} \oplus M^{\prime \prime}$ $\rightarrow M$ that are inverse to each other and provide an isomorphism $M \simeq M^{\prime} \oplus M^{\prime \prime}$ with $f \circ u+v \circ g=i d_{M}$ and thus $\operatorname{ker}(u)=i m(v)$.

These conditions are automatically satisfied if $M^{\prime \prime}$ is free or projective.

Using the notation $M^{*}=\operatorname{hom}_{A}(M, A)$, for any morphism $f: M \rightarrow N$, we 
shall denote by $f^{*}: N^{*} \rightarrow M^{*}$ the morphism which is defined by $f^{*}(h)=$ $h \circ f, \forall h \in h^{\prime} m_{A}(N, A)$ and satisfies $r k_{A}(f)=r k_{A}(i m(f))=r k_{A}\left(f^{*}\right)$, $\forall f \in \operatorname{hom}_{A}(M, N)$ (See [24], Corollary 5.3, p. 179). We may take out $M$ in order to obtain the deleted sequence $\cdots \stackrel{d_{2}}{\rightarrow} F_{1} \stackrel{d_{1}}{\rightarrow} F_{0} \rightarrow 0$ and apply $\operatorname{hom}_{A}(\bullet, A)$ in order to get the sequence $\cdots \stackrel{d_{2}^{*}}{\leftarrow} F_{1}^{*} \stackrel{d_{1}^{*}}{\leftarrow} F_{0}^{*} \leftarrow 0$.

PROPOSITION 4.4: The extension modules $\operatorname{ext}_{A}^{0}(M)=\operatorname{ker}\left(d_{1}^{*}\right)=\operatorname{hom}_{A}(M, A)$ $=M^{*}$ and $\operatorname{ext}^{i}(M)=\operatorname{ext}_{A}^{i}(M)=\operatorname{ker}\left(d_{i+1}^{*}\right) / \operatorname{im}\left(d_{i}^{*}\right), \forall i \geq 1$ do not depend on the resolution chosen and are torsion modules for $i \geq 1$.

Let $A$ be a differential ring, that is a commutative ring with $n$ commuting derivations $\left\{\partial_{1}, \cdots, \partial_{n}\right\}$, that is $\partial_{i} \partial_{j}=\partial_{j} \partial_{i}=\partial_{i j}, \forall i, j=1, \cdots, n$ while $\partial_{i}(a+b)$ $=\partial_{i} a+\partial_{i} b$ and $\partial_{i}(a b)=\left(\partial_{i} a\right) b+a \partial_{i} b, \forall a, b \in A$. We shall use thereafter a differential integral domain $A$ with unit $1 \in A$ whenever we shall need a differential field $\mathbb{Q} \subset K=Q(A)$ of coefficients, that is a field $(a \in K \Rightarrow 1 / a \in K)$ with $\partial_{i}(1 / a)=-\left(1 / a^{2}\right) \partial_{i} a$, in order to exhibit solved forms for systems of partial differential equations as in the preceding section. Using an implicit summation on multi-indices, we may introduce the (noncommutative) ring of differential operators $D=A\left[d_{1}, \cdots, d_{n}\right]=A[d]$ with elements $P=a^{\mu} d_{\mu}$ such that $|\mu|<\infty$ and $d_{i} a=a d_{i}+\partial_{i} a$. The highest value of $|\mu|$ with $a^{\mu} \neq 0$ is called the order of the operator $P$ and the ring $D$ with multiplication $(P, Q) \rightarrow P \circ Q=P Q$ is filtred by the order $q$ of the operators with the filtration $0=D_{-1} \subset D_{0} \subset D_{1} \subset \cdots \subset D_{q} \subset \cdots \subset D_{\infty}=D$. Moreover, it is clear that $D$, as an algebra, is generated by $A=D_{0}$ and $T=D_{1} / D_{0}$ with $D_{1}=A \oplus T$ if we identify an element $\xi=\xi^{i} d_{i} \in T$ with the vector field $\xi=\xi^{i}(x) \partial_{i}$ of differential geometry, but with $\xi^{i} \in A$ now. It follows that $D={ }_{D} D_{D}$ is a bimodule over itself, being at the same time a left $D$-module ${ }_{D} D$ by the composition $P \rightarrow Q P$ and a right $D$-module $D_{D}$ by the composition $P \rightarrow P Q$ with $D_{r} D_{s}=D_{r+s}, \forall r, s \geq 0$ in any case.

If we introduce differential indeterminates $y=\left(y^{1}, \cdots, y^{m}\right)$, we may extend $d_{i} y_{\mu}^{k}=y_{\mu+1_{i}}^{k} \quad$ to $\quad \Phi^{\tau} \equiv a_{k}^{\tau \mu} y_{\mu}^{k} \stackrel{d_{i}}{\rightarrow} d_{i} \Phi^{\tau} \equiv a_{k}^{\tau \mu} y_{\mu+1_{i}}^{k}+\partial_{i} a_{k}^{\tau \mu} y_{\mu}^{k} \quad$ for $\quad \tau=1, \cdots, p$. Therefore, setting $D y^{1}+\cdots+D y^{m}=D y \simeq D^{m}$ and calling $I=D \Phi \subset D y$ the differential module of equations, we obtain by residue the differential module or $D$ - module $M=D y / D \Phi$, introducing the canonical projection $D y \stackrel{p}{\rightarrow} M \rightarrow 0$ and denoting the residue of $y_{\mu}^{k}$ by $\bar{y}_{\mu}^{k}$ when there can be a confusion. Introducing the two free differential modules $F_{0} \simeq D^{m_{0}}, F_{1} \simeq D^{m_{1}}$, we obtain equivalently the free presentation $F_{1} \stackrel{d_{1}}{\rightarrow} F_{0} \stackrel{p}{\rightarrow} M \rightarrow 0$ of order $q$ when $d_{1}=\mathcal{D}=\Phi \circ j_{q}$. It follows that $M$ can be endowed with a quotient filtration obtained from that of $D^{m}$ which is defined by the order of the jet coordinates $y_{q}$ in $D_{q} y$. We shall suppose that the system $R_{q}=\operatorname{ker}(\Phi)$ is formally integrable. We have therefore the inductive limit $0=M_{-1} \subseteq M_{0} \subseteq M_{1} \subseteq \cdots \subseteq M_{q}$ $\subseteq \cdots \subseteq M_{\infty}=M$ with $d_{i} M_{q} \subseteq M_{q+1}$ which is the dual of the projective limit $R=R_{\infty} \rightarrow \cdots \rightarrow R_{q} \rightarrow R_{0} \rightarrow 0$ if we set $R=h \operatorname{mom}_{K}(M, K)$ with

$R_{q}=\operatorname{hom}_{K}\left(M_{q}, K\right)$ and $D R_{q+1} \subseteq T^{*} \otimes R_{q}$, the main reason for using a 
differential field $K$. We have in general $D_{r} I_{s} \subseteq I_{r+s}, \forall r \geq 0, \forall s<q$ with $I_{r}=I \cap D_{r} y$. Also, $R$ is a left $D$-module with $f\left(y_{\mu}^{k}\right)=f_{\mu}^{k}$ and $\left(d_{i} f\right)_{\mu}^{k}=\partial_{i} f_{\mu}^{k}-f_{\mu+1_{i}}^{k}$.

More generally, introducing the successive CC as in the preceding Section while changing slightly the numbering of the respective operators, we may finally obtain the free resolution of $M$, namely the exact sequence $\cdots \stackrel{d_{3}}{\rightarrow} F_{2} \stackrel{d_{2}}{\rightarrow} F_{1}$ $\stackrel{d_{1}}{\rightarrow} F_{0} \stackrel{p}{\rightarrow} M \rightarrow 0$ where $p$ is the canonical projection. Also, with a slight abuse of language, when $D=\Phi \circ j_{q}$ is involutive, that is to say when $R_{q}=\operatorname{ker}(\Phi)$ is involutive, one should say that $M$ has an involutive presentation of order $q$ or that $M_{q}$ is involutive.

REMARK 4.5: In actual practice, one must never forget that $\mathcal{D}=\Phi \circ j_{q}$ acts on the left on column vectors in the operator case and on the right on row vectors in the module case. For this reason, when $E$ is a (finite dimensional) vector bundle over $X$, we may apply the correspondence $J_{\infty}(E) \leftrightarrow D \otimes_{K} E^{*}: J_{q}(E) \leftrightarrow D_{q} \otimes_{K} E^{*} \quad$ with $\quad \pi_{q}^{q+1}: J_{q+1}(E) \rightarrow J_{q}(E) \leftrightarrow D_{q}$ $\subset D_{q+1}$ and $E^{*}=\operatorname{hom}_{K}(E, K)$ between jet bundles and left differential modules in order to be able to use the double dual isomorphism $E \simeq E^{* *}$ in both cases. We shall say that $D(E)=D \otimes_{K} E^{*}=i n d\left(E^{*}\right)$ is the the left differential module induced by $E^{*}$. Hence, starting from a differential operator $E \stackrel{D}{\rightarrow} F$, we may obtain a finite presentation $D \otimes_{K} F^{*} \stackrel{D^{*}}{\rightarrow} D \otimes_{K} E^{*} \stackrel{p}{\rightarrow} M \rightarrow 0$ and conversely, keeping the same operator matrix if we act on the right of row vectors. This comment becomes particularly useful when dealing with the Poincaré sequence in electromagnetism $(n=4)$ or even as we already saw in the Introduction $(n=3)$.

Roughly speaking, homological algebra has been created in order to find intrinsic properties of modules not depending on their presentations or even on their resolutions and we now exhibit another approach by defining the formal adjoint of an operator $P$ and an operator matrix $\mathcal{D}$ :

DEFINITION 4.6: Setting $P=a^{\mu} d_{\mu} \in D \stackrel{a d}{\leftrightarrow} a d(P)=(-1)^{|\mu|} d_{\mu} a^{\mu} \in D$, we have $\operatorname{ad}(\operatorname{ad}(P))=P$ and $\operatorname{ad}(P Q)=a d(Q) a d(P), \forall P, Q \in D$. Such a definition can be extended to any matrix of operators by using the transposed matrix of adjoint operators and we get:

$$
\langle\lambda, \mathcal{D} \xi\rangle=\langle\operatorname{ad}(\mathcal{D}) \lambda, \xi\rangle+\operatorname{div}(\ldots)
$$

from integration by part, where $\lambda$ is a row vector of test functions and \langle\rangle the usual contraction. We quote the useful formulas $r k_{D}(\mathcal{D})=r k_{D}(a d(\mathcal{D}))$ as in ([1] or [5], p. 339-341).

The following technical Lemma is crucially used in the next proposition:

LEMMA 4.7: If $f \in \operatorname{aut}(X)$ is a local diffeomorphisms on $X$, we may set $x=f^{-1}(y)=g(y)$ and we have the identity.

$$
\frac{\partial}{\partial y^{k}}\left(\frac{1}{\Delta(g(y))} \partial_{i} f^{k}(g(y))\right) \equiv 0 .
$$

PROPOSITION 4.8: If we have an operator $E \stackrel{\mathcal{D}}{\rightarrow} F$, we may obtain by 
duality an operator $\wedge^{n} T^{*} \otimes E^{*} \stackrel{\operatorname{ad}(\mathcal{D})}{\leftarrow} \wedge^{n} T^{*} \otimes F^{*}$.

Now, with operational notations, let us consider the two differential sequences:

$$
\begin{gathered}
\xi \stackrel{\mathcal{D}}{\rightarrow} \eta \stackrel{\mathcal{D}_{1}}{\rightarrow} \zeta \\
v \stackrel{\operatorname{ad}(\mathcal{D})}{\leftarrow} \mu \stackrel{a d\left(\mathcal{D}_{1}\right)}{\leftarrow} \lambda
\end{gathered}
$$

where $\mathcal{D}_{1}$ generates all the $\mathrm{CC}$ of $\mathcal{D}$. Then $\mathcal{D}_{1} \circ \mathcal{D} \equiv 0 \Leftrightarrow \operatorname{ad}(\mathcal{D}) \circ \operatorname{ad}\left(\mathcal{D}_{1}\right) \equiv 0$ but $\operatorname{ad}(\mathcal{D})$ may not generate all the CC of $\operatorname{ad}\left(\mathcal{D}_{1}\right)$ as we already saw in the Introduction. Passing to the module framework, we just recognize the definition of $\operatorname{ext}^{1}(M)$ when $M$ is determined by $\mathcal{D}$.

As $D={ }_{D} D_{D}$ is a bimodule, then $M^{*}=\operatorname{hom}_{D}(M, D)$ is a right $D$-module according to Lemma 3.1 and we may thus define a right module $N_{D}$ by the ker/coker long exact sequence $0 \leftarrow N_{D} \leftarrow F_{1}^{*} \stackrel{\mathcal{D}^{*}}{\leftarrow} F_{0}^{*} \leftarrow M^{*} \leftarrow 0$ but we have [11] [31] [46] [47]:

THEOREM 4.9: We have the side changing procedures $M={ }_{D} M \rightarrow M_{D}$ $=\wedge^{n} T^{*} \otimes_{A} M$ and $N_{D} \rightarrow N={ }_{D} N=\operatorname{hom}_{A}\left(\wedge^{n} T^{*}, N_{D}\right)$ with ${ }_{D}\left(M_{D}\right)=M$ and ${ }_{D}\left(N_{D}\right)=N$.

Now, exactly like we defined the differential module $M$ from $\mathcal{D}$, we may define the differential module $N$ from $\operatorname{ad}(\mathcal{D})$. For any other presentation of $M$ with an accent, we have [11] [48]:

THEOREM 4.10: The modules $N$ and $N^{\prime}$ are projectively equivalent, that is one can find two projective modules $P$ and $P^{\prime}$ such that $N \oplus P \simeq N^{\prime} \oplus P^{\prime}$ and we obtain therefore $\operatorname{ext}_{D}^{i}(N) \simeq \operatorname{ext}_{D}^{i}\left(N^{\prime}\right), \forall i \geq 1$.

THEOREM 4.11: The operator $\mathcal{D}$ is simply parametrizable if $\operatorname{ext}^{1}(N)=0$ and doubly parametrizable if $\operatorname{ext}^{1}(N)=0$ and $\operatorname{ext}^{2}(N)=0$. Moreover, we have the ker/coker long exact sequence:

$$
0 \rightarrow \operatorname{ext}^{1}(N) \rightarrow M \stackrel{\epsilon}{\rightarrow} M^{* *} \rightarrow \operatorname{ext}^{2}(N) \rightarrow 0
$$

where $(\epsilon(m))(f)=f(m)$ whenever $f \in M^{*}$ and we have $t(M)=\operatorname{ext}^{1}(N)=$ $\operatorname{ker}(\epsilon)$.

Proof: We prove first that $t(M) \subseteq \operatorname{ker}(\epsilon)$. Indeed, if $m \in t(M)$, then one may find $0 \neq P \in D$ such that $P m=0$ and thus $f(P m)=P f(m)=0$ $\Rightarrow f(m)=0$ because $D=K[d]$ is an integral domain and thus $t(M) \subseteq \operatorname{ker}(\epsilon)$.

Let us now start with a free presentation of $M=\operatorname{cocker}\left(d_{1}\right)$ :

$$
F_{1} \stackrel{d_{1}}{\rightarrow} F_{0} \stackrel{p}{\rightarrow} M \rightarrow 0
$$

Applying $\operatorname{hom}_{D}(M, D)$, we may define $N_{D}=\operatorname{coker}\left(d_{1}^{*}\right)$ and exhibit the following free resolution of $N$ by right $D$-modules:

$$
0 \leftarrow N_{D} \leftarrow F_{1}^{*} \stackrel{d_{1}^{*}}{\leftarrow} F_{0}^{*} \stackrel{d_{0}^{*}}{\leftarrow} F_{-1}^{*} \stackrel{d_{-1}^{*}}{\leftarrow} F_{-2}^{*}
$$

where $M^{*}=\operatorname{ker}\left(d_{1}^{*}\right)=\operatorname{im}\left(d_{0}^{*}\right) \simeq \operatorname{coker}\left(d_{-1}^{*}\right)$. The deleted sequence is:

$$
0 \leftarrow F_{1}^{*} \stackrel{d_{1}^{*}}{\leftarrow} F_{0}^{*} \stackrel{d_{0}^{*}}{\leftarrow} F_{-1}^{*} \stackrel{d_{-1}^{*}}{\leftarrow} F_{-2}^{*}
$$


Applying again $\operatorname{hom}_{D}(\bullet, D)$ and using the canonical isomorphism $F^{* *} \simeq F$ for any free module $F$ of finite rank, we get the sequence of left $D$-modules:

$$
\begin{aligned}
& 0 \rightarrow F_{1} \stackrel{d_{1}}{\rightarrow} F_{0} \stackrel{d_{0}}{\rightarrow} \quad F_{-1} \stackrel{\stackrel{d_{-1}}{\rightarrow}}{\rightarrow} F_{-2} \\
& \downarrow \searrow \uparrow \\
& \begin{array}{ccc}
M & \stackrel{\epsilon}{\rightarrow} & M^{* *} \\
\downarrow & & \uparrow
\end{array} \\
& 0 \quad 0
\end{aligned}
$$

Denoting as usual a coboundary space by $B$, a cocycle space by $Z$ and the corresponding cohomology by $H=Z / B$, we get the commutative and exact diagram:

$$
\begin{aligned}
& 0 \rightarrow B_{0} \rightarrow F_{0} \rightarrow M \quad \rightarrow 0 \\
& \downarrow \quad \| \quad \downarrow \epsilon \\
& 0 \rightarrow Z_{0} \rightarrow F_{0} \rightarrow M^{* *}
\end{aligned}
$$

An easy chase provides at once $H_{0}=Z_{0} / B_{0}=\operatorname{ext}_{D}^{1}(N) \simeq \operatorname{ker}(\epsilon)$. It follows that $\operatorname{ker}(\epsilon)$ is a torsion module and, as we already know that $t(M) \subseteq \operatorname{ker}(\epsilon)$ $\subseteq M$, we finally obtain $t(M)=\operatorname{ker}(\epsilon)$. Also, as $B_{-1}=\operatorname{im}(\epsilon)$ and $Z_{-1} \simeq M^{* *}$, we obtain $H_{-1}=Z_{-1} / B_{-1}=\operatorname{ext}_{A}^{2}(N, A) \simeq \operatorname{coker}(\epsilon)$. Accordingly, a torsion-free ( $\epsilon$ injective)/reflexive ( $\epsilon$ bijective) module is described by an operator that admits respectively a single/double step parametrization.

Q.E.D.

We now turn to the operator framework;

DEFINITION 4.12: If a differential operator $\xi \stackrel{\mathcal{D}}{\rightarrow} \eta$ is given, a direct problem is to find generating compatibility conditions (CC) as an operator $\eta \stackrel{\mathcal{D}_{1}}{\rightarrow} \zeta$ such that $D \xi=\eta \Rightarrow D_{1} \eta=0$. Conversely, given $\eta \stackrel{\mathcal{D}_{1}}{\rightarrow} \zeta$, the inverse problem will be to look for $\xi \stackrel{\mathcal{D}}{\rightarrow} \eta$ such that $\mathcal{D}_{1}$ generates the CC of $\mathcal{D}$ and we shall say that $\mathcal{D}_{1}$ is parametrized by $\mathcal{D}$ if such an operator $\mathcal{D}$ is existing. We finally notice that any operator is the adjoint of a certain operator because $\operatorname{ad}(\operatorname{ad}(P))=P, \forall P \in D$ and we get:

THEOREM 4.13: (reflexivity test) In order to check whether $M$ is reflexive or not, that is to find out a parametrization if $t(M)=0$ which can be again parametrized, the test has 5 steps which are drawn in the following diagram where $\operatorname{ad}(\mathcal{D})$ generates the CC of $a d\left(\mathcal{D}_{1}\right)$ and $\mathcal{D}_{1}^{\prime}$ generates the CC of $\mathcal{D}=\operatorname{ad}(\operatorname{ad}(\mathcal{D}))$ while $\operatorname{ad}\left(\mathcal{D}_{-1}\right)$ generates the $\mathrm{CC}$ of $\operatorname{ad}(\mathcal{D})$ and $\mathcal{D}^{\prime}$ generates the CC of $\mathcal{D}_{-1}$ :

$$
\begin{aligned}
& \begin{array}{lllll} 
& \eta^{\prime} & & \zeta^{\prime} & 5 \\
\mathcal{D}^{\prime} & & \mathcal{D}_{1}^{\prime} & & \\
\nearrow & & \nearrow &
\end{array} \\
& 4 \quad \phi \quad \stackrel{\mathcal{D}_{-1}}{\rightarrow} \xi \quad \stackrel{\mathcal{D}}{\rightarrow} \quad \eta \quad \stackrel{\mathcal{D}_{1}}{\rightarrow} \quad \zeta \quad 1 \\
& 3 \quad \theta \stackrel{a d\left(\mathcal{D}_{-1}\right)}{\leftarrow} \quad v \stackrel{a d(\mathcal{D})}{\leftarrow} \mu \stackrel{a d\left(\mathcal{D}_{1}\right)}{\leftarrow} \lambda \quad \lambda \quad 2
\end{aligned}
$$


$\mathcal{D}_{1}$ parametrized by $\mathcal{D} \Leftrightarrow \mathcal{D}_{1}=\mathcal{D}_{1}^{\prime} \Leftrightarrow \operatorname{ext}^{1}(N)=0 \Leftrightarrow \epsilon$ injective $\Leftrightarrow t(M)=0$

$\mathcal{D}$ parametrized by $\mathcal{D}_{-1} \Leftrightarrow \mathcal{D}=\mathcal{D}^{\prime} \Leftrightarrow \operatorname{ext}^{2}(N)=0 \Leftrightarrow \epsilon$ surjective

COROLLARY 4.14: In the differential module framework, if $F_{1} \stackrel{D_{1}}{\rightarrow} F_{0} \stackrel{p}{\rightarrow} M$ $\rightarrow 0$ is a finite free presentation of $M=\operatorname{coker}\left(\mathcal{D}_{1}\right)$ with $t(M)=0$, then we may obtain an exact sequence $F_{1} \stackrel{\mathcal{D}_{1}}{\rightarrow} F_{0} \stackrel{\mathcal{D}}{\rightarrow} E$ of free differential modules where $\mathcal{D}$ is the parametrizing operator. However, there may exist other parametrizations $F_{1} \stackrel{\mathcal{D}_{1}}{\rightarrow} F_{0} \stackrel{\mathcal{D}^{\prime}}{\rightarrow} E^{\prime}$ called minimal parametrizations such that $\operatorname{coker}\left(\mathcal{D}^{\prime}\right)$ is a torsion module and we have thus $r k_{D}(M)=r k_{D}\left(E^{\prime}\right)$.

REMARK 4.15: The following chains of inclusions and short exact sequences allow to compare the main procedures used in the respective study of differential extensions and differential modules:

$$
\begin{aligned}
& K \subset K\langle S\rangle \subset L \Rightarrow 0 \rightarrow F \rightarrow M \rightarrow T \rightarrow 0 \\
& K \subset K^{\prime} \subset L \Rightarrow 0 \rightarrow t(M) \rightarrow M \rightarrow M^{\prime} \rightarrow 0
\end{aligned}
$$

where $F$ is a maximum free submodule of $M, T=M / F$ is a torsion-module and $M^{\prime}=M / t(M)$ is a torsion-free module. The next examples open the way towards a new domain of research.

EXAMPLE 4.16: With $n=2, m=3, K=\mathbb{Q}$, let us consider the first order nonlinear involutive system:

$$
P_{1} \equiv y_{2}^{1}-y^{3} y_{1}^{1}=0, \quad P_{2} \equiv y_{2}^{2}-y^{3} y_{1}^{2}=0
$$

This system defines a prime differential ideal $\mathfrak{p} \subset K\{y\}$ and the differential extension $L=Q(K\{y\} / \mathfrak{p})$ is differentially algebraic over $K\left\langle y^{3}\right\rangle$ with parametric jets $\left(y^{1}, y^{2}, y_{1}^{1}, y_{1}^{2}, y_{11}^{1}, y_{11}^{2}, \cdots\right)$.

The linearized system $\mathcal{D}_{1} Y=0$ over $L$ is:

$$
d_{2} Y^{1}-y^{3} d_{1} Y^{1}-y_{1}^{1} Y^{3}=0, \quad d_{2} Y^{2}-y^{3} d_{1} Y^{2}-y_{1}^{2} Y^{3}=0
$$

Multiplying by test functions $\left(\lambda^{1}, \lambda^{2}\right)$ and integrating by part, we get $\operatorname{ad}\left(\mathcal{D}_{1}\right) \lambda=\mu$ in the form:

$$
\left\{\begin{array}{l}
Y^{1} \rightarrow-d_{2} \lambda^{1}+y^{3} d_{1} \lambda^{1}+y_{1}^{3} \lambda^{1}=\mu^{1} \\
Y^{2} \rightarrow-d_{2} \lambda^{2}+y^{3} d_{1} \lambda^{2}+y_{1}^{3} \lambda^{2}=\mu^{2} \\
Y^{3} \rightarrow-y_{1}^{1} \lambda^{1}-y_{1}^{2} \lambda^{2}=\mu^{3}
\end{array}\right.
$$

Using only the parametric jets for $y$ and $\lambda$ in the PD equations provided, we get:

$$
\begin{aligned}
& -y_{1}^{1}\left(y^{3} d_{1} \lambda^{1}+y_{1}^{3} \lambda^{1}\right)-\left(y^{3} y_{11}^{1}+y_{1}^{1} y_{1}^{3}\right) \lambda^{1}-y_{1}^{2}\left(y^{3} d_{1} \lambda_{2}+y_{1}^{3} \lambda^{1}\right)-\left(y^{3} y_{11}^{2}+y_{1}^{2} y_{1}^{3}\right) \lambda^{2} \\
& =d_{2} \mu^{3}-y_{1}^{1} \mu^{1}-y_{1}^{2} \mu^{2} \\
& \quad-y^{3} y_{1}^{1} d_{1} \lambda^{1}-y^{3} y_{11}^{1} \lambda^{1}-y^{3} y_{1}^{2} d_{1} \lambda^{2}-y^{3} y_{11}^{2} \lambda^{2}-2 y_{1}^{1} y_{1}^{3} \lambda^{1}-2 y_{1}^{2} y_{1}^{3} \lambda^{2} \\
& =y^{3} d_{1} \mu^{3}+2 y_{1}^{3} \mu^{3}
\end{aligned}
$$

and the onlyCC $\operatorname{ad}(\mathcal{D}) \mu=0$ over $L$ :

$$
-d_{2} \mu^{3}+y^{3} d_{1} \mu^{3}+y_{1}^{1} \mu^{1}+y_{1}^{2} \mu^{2}+2 y_{1}^{3} \mu^{3}=0
$$


Multiplying by a test function $\xi$ and integrating by part, we get $\mathcal{D} \xi=Y$ over $L$ in the form:

$$
y_{1}^{1} \xi=Y^{1}, y_{1}^{2} \xi=Y^{2}, d_{2} \xi-y^{3} d_{1} \xi+y_{1}^{3} \xi=Y^{3}
$$

admitting the $\mathrm{CC} \mathcal{D}_{1} Y=0$ of course but also the additional zero order CC:

$$
\omega \equiv y_{1}^{1} Y^{2}-y_{1}^{2} Y^{1}=0
$$

which provides a torsion element $\omega$ satisfying $d_{2} \omega-y^{3} d_{1} \omega-y_{1}^{3} \omega=0$. Setting $Y=\delta y$ as the standard variational notation used by engineers, we obtain easily $\omega \wedge \delta \omega \neq 0$ and $\omega$ cannot therefore admit an integrating factor, a result showing that $K$ is its own differential algebraic closure in $L$.

EXAMPLE 4.17: If $\alpha=d x^{1}-x^{3} d x^{2} \in T^{*}$, the linear system obtained over $K=\mathbb{Q}\left(x^{1}, x^{2}, x^{3}\right)$ by eliminating the factor $\rho(x)$ in the linear system $\mathcal{L}(\xi) \alpha=\rho(x) \alpha$ admits the injective parametrization $-x^{3} \partial_{3} \phi+\phi=\xi^{1}$, $-\partial_{3} \phi=\xi^{2}, \quad \partial_{2} \phi-x^{3} \partial_{1} \phi=\xi^{3} \Rightarrow \xi^{1}-x^{3} \xi^{2}=\phi$. It defines therefore a free differential module $M \simeq D$ which is thus reflexive and even projective. Any resolution of this module splits, like the short exact sequence $0 \rightarrow D^{2} \rightarrow D^{3}$ $\rightarrow D \rightarrow 0$, and the corresponding differential sequence of operators is locally exact like the Poincaré sequence ([3], p. 684-691).

\section{Applications}

We start this section with a general (difficult) result on the actions of Lie groups, covering at the same time the study of the classical and conformal Killing systems. For this, we notice that the involutive first Spencer operator $D_{1}: C_{0}=R_{q}$ $\stackrel{j_{1}}{\rightarrow} J_{1}\left(R_{q}\right) \rightarrow J_{1}\left(R_{q}\right) / R_{q+1} \simeq T^{*} \otimes R_{q} / \delta\left(g_{q+1}\right)=C_{1}$ of order one is induced by the Spencer operator $D: R_{q+1} \rightarrow T^{*} \otimes R_{q}: \xi_{q+1} \rightarrow j_{1}\left(\xi_{q}\right)-\xi_{q+1}=\left\{\partial_{i} \xi_{\mu}^{k}-\xi_{\mu+1_{i}}^{k}|0 \leq| \mu \mid q\right\}$. Introducing the Spencer bundles $C_{r}=\wedge^{r} T^{*} \otimes R_{q} / \delta\left(\wedge^{r-1} T^{*} \otimes g_{q+1}\right)$, the first order involutive $(r+1)$-Spencer operator $D_{r+1}: C_{r} \rightarrow C_{r+1}$ is induced by $D: \wedge^{r} T^{*} \otimes R_{q+1} \rightarrow \wedge^{r+1} T^{*} \otimes R_{q}: \alpha \otimes \xi_{q+1} \rightarrow d \alpha \otimes \xi_{q}+(-1)^{r} \alpha \wedge D \xi_{q+1}$. We obtain therefore the canonical linear Spencer sequence ([5], p. 150 or [38]) (See [49] [50] [51] [52] for other applications):

$$
0 \rightarrow \Theta \stackrel{j_{q}}{\rightarrow} C_{0} \stackrel{D_{1}}{\rightarrow} C_{1} \stackrel{D_{2}}{\rightarrow} C_{2} \stackrel{D_{3}}{\rightarrow} \cdots \stackrel{D_{n}}{\rightarrow} C_{n} \rightarrow 0
$$

PROPOSITION 5.1: The Spencer sequence for the Lie operator describing the infinitesimal action of a Lie group $G$ is (locally) isomorphic to the tensor product of the Poincaré sequence by the Lie algebra $\mathcal{G}=T_{e}(G)$ where $e \in G$ is the identity element. It follows that $D_{r+1}$ generates the CC of $D_{r} \Leftrightarrow \operatorname{ad}\left(D_{r}\right)$ generates the CC of ad $\left(D_{r+1}\right)$, a result not evident at all.

Proof: We may introduce a basis $\left\{\theta_{\tau}=\theta_{\tau}^{i}(x) \partial_{i}\right\}$ of infinitesimal generators of the action with $\tau=1, \cdots, \operatorname{dim}(G)$ and the commutation relations $\left[\theta_{\rho}, \theta_{\sigma}\right]=$ $c_{\rho \sigma}^{\tau} \theta_{\tau}$ discovered by S. Lie giving the structure constants $c$ of $\mathcal{G}$ (See [34] and [44] for more details). Any element $\lambda \in \mathcal{G}$ can be written $\lambda=\left\{\lambda^{\tau}=c s t\right\}$. "Gauging" such an element, that is to say replacing the constants by functions or, equivalently, introducing a map $X \rightarrow \wedge^{0} T^{*} \otimes \mathcal{G}:(x) \rightarrow\left(\lambda^{\tau}(x)\right)$, we may obtain 
locally a map $\wedge^{0} T^{*} \otimes \mathcal{G} \rightarrow T: \lambda^{\tau}(x) \rightarrow \lambda^{\tau}(x) \theta_{\tau}^{k}(x)$ or, equivalently, vector fields $\xi=\left(\xi^{i}(x) \partial_{i}\right) \in T$ of the form $\xi^{k}(x)=\lambda^{\tau}(x) \theta_{\tau}^{k}(x)$, keeping the index $i$ for 1-forms. More generally, we can introduce a map:

$$
\begin{aligned}
& \wedge^{r} T^{*} \otimes \mathcal{G} \rightarrow \wedge^{r} T^{*} \otimes J_{q}(T)=\lambda \rightarrow \lambda \otimes j_{q}(\theta)=X_{q}: \lambda^{\tau}(x) \\
& \rightarrow \lambda^{\tau}(x) \partial_{\mu} \theta_{\tau}^{k}(x)=X_{\mu, I}^{k}(x) d x^{I}
\end{aligned}
$$

that we can lift to the element $\lambda \otimes j_{q+1}(\theta)=X_{q+1} \in \wedge^{r} T^{*} \otimes J_{q+1}(T)$. It follows from the definitions that $D_{r} X_{q}=D X_{q+1}$ by introducing any element of $C_{r}(T)$ through its representative $X_{q} \in \wedge^{r} T^{*} \otimes J_{q}(T)$. We obtain therefore the crucial formula:

$$
\begin{aligned}
D_{r} X_{q} & =D X_{q+1} \\
& =D\left(\lambda \otimes j_{q+1}(\theta)\right) \\
& =d \lambda \otimes j_{q}(\theta)+(-1)^{r} \lambda \wedge D j_{q+1}(\theta) \\
& =d \lambda \otimes j_{q}(\theta)
\end{aligned}
$$

allowing to identify locally the Spencer sequence with a tensor product of the Poincaré sequence, because $g_{q}=0 \Rightarrow C_{r}=\wedge^{r} T^{*} \otimes R_{q}$. When the action is effective, the map $\wedge^{0} T^{*} \otimes \mathcal{G} \rightarrow J_{q}(T)$ is injective. We obtain therefore an isomorphism $\wedge^{0} T^{*} \otimes \mathcal{G} \rightarrow R_{q} \subset J_{q}(T)$ when $q$ is large enough allowing to exhibit an isomorphism between the canonical Spencer sequence and the tensor product of the Poincaré sequence by $\mathcal{G}$ when $q$ is large enough in such a way that $R_{q}$ is involutive with $\operatorname{dim}\left(R_{q}\right)=\operatorname{dim}(\mathcal{G})$ and $g_{q}=0$.

Q.E.D.

We now study what happens when $n \geq 3$ because the case $n=2$ has already been provided, proving that conformal geometry must be entirely revisited.

- $n=3$ : Using the euclidean metric $\omega$, we have 6 components of $\Omega \in F_{0}$ $=S_{2} T^{*}$ with $\operatorname{dim}\left(F_{0}\right)=n(n+1) / 2=6$ in the case of the classical Killing system/operator and obtain easily the $n^{2}\left(n^{2}-1\right) / 12=6$ components of the second order Riemann operator, linearization of the Riemann tensor at $\omega$. We have $n^{2}\left(n^{2}-1\right)(n-2) / 24=3$ first order Bianchi identities ([3], p. 625). Introducing the respective adjoint operators while taking into account the last Proposition and the fact that the extension modules do not depend on the resolution used (a difficult result indeed!), we get the following diagram where we have set $a d($ Riemann $)=$ Beltrami for historical reasons [30] and each operator generates the $\mathrm{CC}$ of the next one:

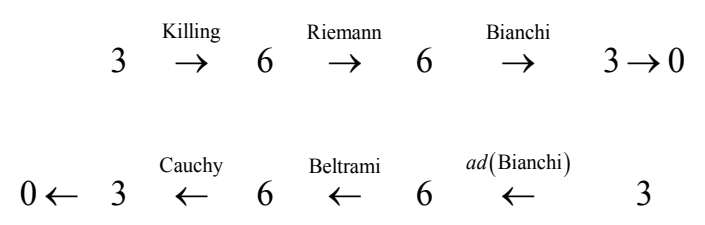

As in the Introduction where Airy $=a d($ Riemann $)$, the Beltrami operator is now parametrizing the 3 Cauchy stress equations [30] but it is rather striking to discover 
that the central second order operator is self-adjoint and can be given as follows:

$$
\left(\begin{array}{cccccc}
0 & 0 & 0 & d_{33} & -2 d_{23} & d_{22} \\
0 & -2 d_{33} & 2 d_{23} & 0 & 2 d_{13} & -2 d_{12} \\
0 & 2 d_{23} & -2 d_{22} & -2 d_{13} & 2 d_{12} & 0 \\
d_{33} & 0 & -2 d_{13} & 0 & 0 & d_{11} \\
-2 d_{23} & 2 d_{13} & 2 d_{12} & 0 & -2 d_{11} & 0 \\
d_{22} & -2 d_{12} & 0 & d_{11} & 0 & 0
\end{array}\right)
$$

The study of the conformal case is much more delicate. As $\hat{F}_{0}$ can be described by trace-free symmetric tensors, we have $\operatorname{dim}\left(\hat{F}_{0}\right)=\operatorname{dim}\left(F_{0}\right)-1=5$ and it remains to discover the operator that will replace the Riemann operator. Having in mind the diagram of Proposition 2.11 and the fact that $\operatorname{dim}\left(\hat{g}_{2}\right)=3$ while $\hat{g}_{3}=0 \Rightarrow \hat{g}_{4}=0$, we have successively:

- NO CC order 1:

$0 \rightarrow \hat{g}_{2} \rightarrow S_{2} T^{*} \otimes T \rightarrow T^{*} \otimes \hat{F}_{0} \rightarrow \hat{F}_{1} \Rightarrow 0 \Rightarrow \operatorname{dim}\left(\hat{F}_{1}\right)=3-18+15=0$.

- NO CC order 2:

$0 \rightarrow \hat{g}_{3} \rightarrow S_{3} T^{*} \otimes T \rightarrow S_{2} T^{*} \otimes \hat{F}_{0} \rightarrow \hat{F}_{1} \Rightarrow 0 \Rightarrow \operatorname{dim}\left(\hat{F}_{1}\right)=0-30+30=0$.

- OK CC order 3:

$0 \rightarrow \hat{g}_{4} \rightarrow S_{4} T^{*} \otimes T \rightarrow S_{3} T^{*} \otimes \hat{F}_{0} \rightarrow \hat{F}_{1} \Rightarrow 0 \Rightarrow \operatorname{dim}\left(\hat{F}_{1}\right)=0-45+50=5$.

Once again, the central third order operator is self-adjoint as can be easily seen by proving that the last $5 \rightarrow 3$ operator, obtained in [6] by means of computer algebra, can be chosen to be the transpose of the first $3 \rightarrow 5$ conformal Killing operator, just by changing columns.

This result can also be obtained by using the fact that, when an operator/a system is formally integrable, the order of the generating CC is equal to the number of prolongations needed to get a 2 -acyclic symbol plus 1 ([5], p. 120, [6]). In the present case, neither $\hat{g}_{1}$ nor $\hat{g}_{2}$ are 2-acyclic while $\hat{g}_{3}=0$ is trivially involutive, so that $(3-1)+1=3$.

- $n=4$ : In the classical case, we may proceed as before for exibiting the 20 components of the second order Riemann operator and the 20 components of the first order Bianchi operator.

The study of the conformal case is much more delicate and still unknown. Indeed, the symbol $\hat{g}_{2}$ is 2 -acyclic when $n \geq 4$ and 3-acyclic when $n \geq 5$. Accordingly, the Weyl operator, namely the CC for the conformal Killing operator, is second order like the Riemann operator. However, when $n=4$ only (care), the symbol $\hat{h}_{2}$ of the Weyl system is not 2-acyclic while its first prolongation $\hat{h}_{3}$ becomes 2-acyclic. It follows that the CC for the Weyl operator are second order, ... and so on. For example, we have the long exact sequence:

$$
0 \rightarrow \hat{g}_{5} \rightarrow S_{5} T^{*} \otimes T \rightarrow S_{4} T^{*} \otimes \hat{F}_{0} \rightarrow S_{2} T^{*} \otimes \hat{F}_{1} \rightarrow \hat{F}_{2} \rightarrow 0
$$

and deduce that $\operatorname{dim}\left(\hat{F}_{2}\right)=(-0)+(56 \times 4)-(35 \times 9)+(10 \times 10)=9$, a result that can be ckecked by computer algebra in a few milliseconds but is still unknown.

We shall finally prove below that the Einstein parametrization of the stress 
equations is neither canonical nor minimal in the following diagrams:

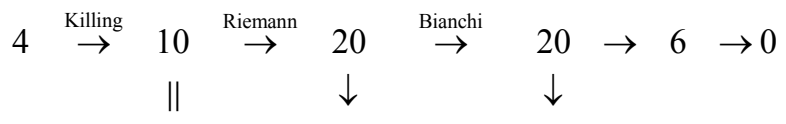

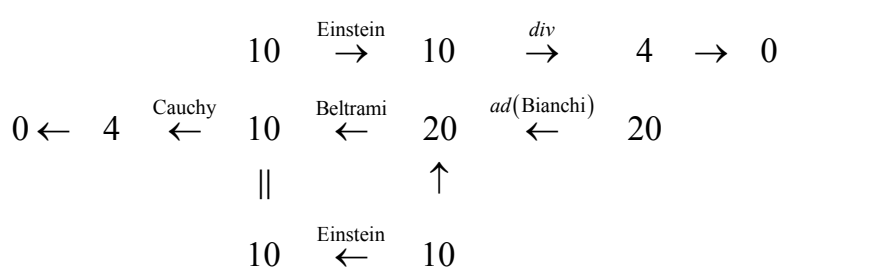

obtained by using the fact that the Einstein operator is self-adjoint, where by Einstein operator we mean the linearization of the Einstein equations at the Minkowski metric, the 6 terms being exchanged between themselves [1] [52]. Indeed, setting $E_{i j}=R_{i j}-\frac{1}{2} \omega_{i j} t r(R)$ with $\operatorname{tr}(R)=\omega^{i j} R_{i j}$, it is essential to notice that the Ricci operator is not self-adjoint because we have for example:

$$
\lambda^{i j}\left(\omega^{r s} d_{i j} \Omega_{r s}\right) \stackrel{a d}{\rightarrow}\left(\omega^{r s} d_{i j} \lambda^{i j}\right) \Omega_{r s}
$$

and ad provides a term appearing in $-\omega_{i j} t r(R)$ but not in $2 R_{i j}$ because we have, as in (5.1.4) of [12]:

$$
\operatorname{tr}(\Omega)=\omega^{r s} \Omega_{r s} \Rightarrow \operatorname{tr}(R)=\omega^{r s} d_{r s} \operatorname{tr}(\Omega)-d_{r s} \Omega^{r s}
$$

The upper div induced by Bianchi has nothing to do with the lower Cauchy stress equations, contrary to what is still believed today while the 10 on the right of the lower diagram has nothing to do with the perturbation of a metric which is the 10 on the left in the upper diagram. It also follows that the Einstein equations in vacuum cannot be parametrized as we have the following diagram of operators recapitulating the five steps of the parametrizability criterion (See [1] [24] for more details or [6] [25] for a computer algebra exhibition of this result):

$$
\begin{array}{ccccc} 
& & \substack{\nearrow \\
\text { Riemann }} & 20 \\
& \stackrel{\text { Killing }}{\rightarrow} & 10 & \stackrel{\text { Einstein }}{\rightarrow} & 10 \\
4 & \stackrel{\text { Cauchy }}{\leftarrow} & 10 & \stackrel{\text { Einstein }}{\leftarrow} & 10
\end{array}
$$

We are facing only two possibilities, both leading to a contradiction:

1) If we use the operator $S_{2} T^{*} \stackrel{\text { Einstein }}{\rightarrow} S_{2} T^{*}$ in the geometrical setting, the $S_{2} T^{*}$ on the left has indeed someting to do with the perturbation of the metric but the $S_{2} T^{*}$ on the right has nothing to do with the stress.

2) If we use the adjoint operator $\wedge^{n} T^{*} \otimes S_{2} T \stackrel{\text { Einstein }}{\leftarrow} \wedge^{n} T^{*} \otimes S_{2} T$ in the physical setting, then $\wedge^{n} T^{*} \otimes S_{2} T$ on the left has of course something to do with the stress but the $\wedge^{n} T^{*} \otimes S_{2} T$ on the right has nothing to do with the perturbation of a metric.

These purely mathematical results question the origin and existence of 
gravitational waves.

We may summarize these results, which do not seem to be known, by the following differential sequences where the order of an operator is written under its arrow:

- $n=3: 3 \rightarrow 5 \rightarrow 5 \rightarrow 3 \rightarrow 0$

- $n=4: 4 \rightarrow 9 \rightarrow 10 \rightarrow 9 \rightarrow 4 \rightarrow 0$

- $n=5: 5 \underset{1}{\rightarrow} 14 \underset{2}{\rightarrow} 35 \underset{1}{\rightarrow} 35 \underset{2}{\rightarrow} 14 \underset{1}{\rightarrow} 5 \rightarrow 0$

THEOREM 5.2: Recalling that we have $g_{2}=0 \Rightarrow g_{3}=0, \hat{g}_{3}=0$ and thus:

$$
F_{1}=H^{2}\left(g_{1}\right)=Z^{2}\left(g_{1}\right), \quad \hat{F}_{1}=H^{2}\left(\hat{g}_{1}\right)=Z^{2}\left(\hat{g}_{1}\right) / \delta\left(T^{*} \otimes \bar{g}_{2}\right)
$$

we have the following commutative and exact "fundamental diagram IP":

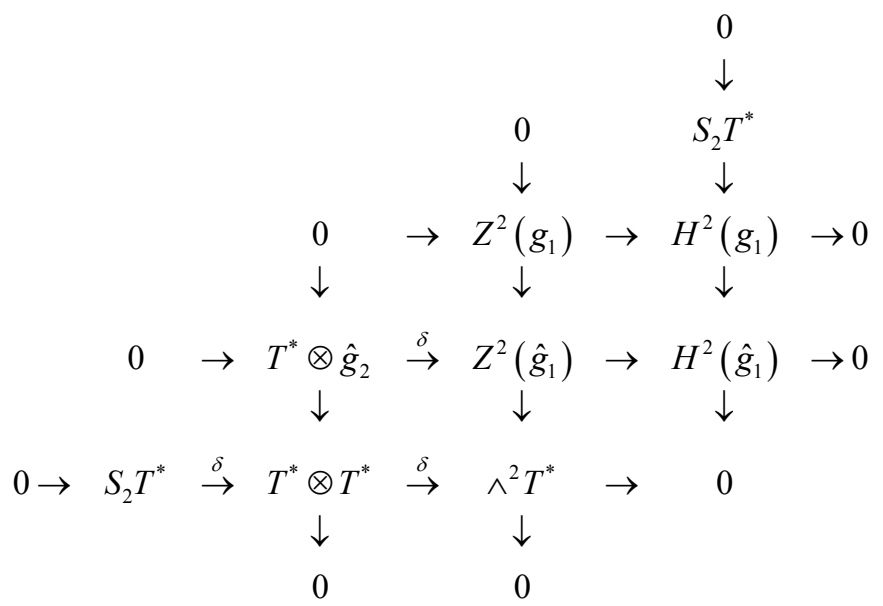

The following theorem will provide all the classical formulas of both Riemannian and conformal geometry in one piece but in a totally unusual framework not depending on any conformal factor.

THEOREM 5.3: All the short exact sequences of the preceding diagram split in a canonical way, that is in a way compatible with the underlying tensorial properties of the vector bundles involved.

$$
\begin{aligned}
& T^{*} \otimes T^{*} \simeq S_{2} T^{*} \oplus \wedge^{2} T^{*} \\
& \Rightarrow Z^{2}\left(\hat{g}_{1}\right)=Z^{2}\left(g_{1}\right)+\delta\left(T^{*} \otimes \hat{g}_{2}\right) \simeq Z^{2}\left(g_{1}\right) \oplus \wedge^{2} T^{*} \\
& \Rightarrow H^{2}\left(g_{1}\right) \simeq H^{2}\left(\hat{g}_{1}\right) \oplus S_{2} T^{*}
\end{aligned}
$$

Proof: First of all, we recall that:

$$
\begin{gathered}
g_{1}=\left\{\xi_{i}^{k} \in T^{*} \otimes T \mid \omega_{r j} \xi_{i}^{r}+\omega_{i r} \xi_{j}^{r}=0\right\} \\
\subset \hat{g}_{1}=\left\{\xi_{i}^{k} \in T^{*} \otimes T \mid \omega_{r j} \xi_{i}^{r}+\omega_{i r} \xi_{j}^{r}-\frac{2}{n} \omega_{i j} \xi_{r}^{r}=0\right\} \\
\Rightarrow \quad 0=g_{2} \subset \hat{g}_{2}=\left\{\xi_{i j}^{k} \in S_{2} T^{*} \otimes T \mid n \xi_{i j}^{k}=\delta_{i}^{k} \xi_{r j}^{r}+\delta_{j}^{k} \xi_{r i}^{r}-\omega_{i j} \omega^{k s} \xi_{r s}^{r}\right\}
\end{gathered}
$$

Now, if $\left(\tau_{l i, j}^{k}\right) \in T^{*} \otimes \hat{g}_{2}$, then we have:

$$
n \tau_{l i, j}^{k}=\delta_{l}^{k} \tau_{r i, j}^{r}+\delta_{i}^{k} \tau_{r l, j}^{r}-\omega_{l i} \omega^{k s} \tau_{r s, j}^{r}
$$

and we may set $\tau_{r i, j}^{r}=\tau_{i, j} \neq \tau_{j, i}$ with $\left(\tau_{i, j}\right) \in T^{*} \otimes T$ and such a formula does 
not depend on any conformal factor [53]. We have:

$$
\delta\left(\tau_{l i, j}^{k}\right)=\left(\tau_{l i, j}^{k}-\tau_{l j, i}^{k}\right)=\left(\rho_{l, i j}^{k}\right) \in B^{2}\left(\hat{g}_{1}\right) \subset Z^{2}\left(\hat{g}_{1}\right)
$$

with:

$$
\begin{gathered}
Z^{2}\left(\hat{g}_{1}\right)=\left\{\left(\rho_{l, i j}^{k}\right) \in \wedge^{2} T^{*} \otimes \hat{g}_{1} \mid \delta\left(\rho_{l, i j}^{k}\right)=0\right\} \Rightarrow \varphi_{i j}=\rho_{r, i j}^{r} \neq 0 \\
\delta\left(\rho_{l, j i}^{k}\right)=\left(\sum_{(l, i, j)} \rho_{l, i j}^{k}=\rho_{l, i j}^{k}+\rho_{i, j l}^{k}+\rho_{j, l i}^{k}\right) \in \wedge^{3} T^{*} \otimes T
\end{gathered}
$$

- The splitting of the lower row is obtained by setting $\left(\tau_{i, j}\right) \in T^{*} \otimes T^{*}$ $\overrightarrow{1}\left(\frac{1}{2}\left(\tau_{i, j}+\tau_{j, i}\right)\right) \in S_{2} T^{*}$ in such a way that $\left(\tau_{i, j}=\tau_{j, i}=\tau_{i j}\right) \in S_{2} T^{*} \Rightarrow$ $\frac{1}{2}\left(\tau_{i j}+\tau_{j i}\right)=\tau_{i j}$.

Similarly, $\left(\varphi_{i j}=-\varphi_{j i}\right) \in \wedge^{2} T^{*} \rightarrow\left(\frac{1}{2} \varphi_{i j}\right) \in T^{*} \otimes T^{*}$ and $\left(\frac{1}{2} \varphi_{i j}-\frac{1}{2} \varphi_{j i}\right)=\left(\varphi_{i j}\right)$ $\in \wedge^{2} T^{*}$.

- The most important result is to split the right column. For this, we first need to describe the monomorphism $0 \rightarrow S_{2} T^{*} \rightarrow H^{2}\left(g_{1}\right)$ which is in fact produced by a diagonal north-east snake type chase. Let us choose $\left(\tau_{i j}=\tau_{i, j}=\tau_{j, i}=\tau_{j i}\right) \in S_{2} T^{*} \subset T^{*} \otimes T^{*}$. Then, we may find $\left(\tau_{l i, j}^{k}\right) \in T^{*} \otimes \hat{g}_{2}$ by deciding that $\tau_{r i, j}^{r}=\tau_{i, j}=\tau_{j, i}=\tau_{r j, i}^{r}$ in $Z^{2}\left(\hat{g}_{1}\right)$ and apply $\delta$ in order to get $\rho_{l, i j}^{k}=\tau_{l i, j}^{k}-\tau_{k, j, i}^{k}$ such that $\rho_{r, i j}^{r}=\varphi_{i j}=0$ and thus $\left(\rho_{l, i j}^{k}\right) \in Z^{2}\left(g_{1}\right)=$ $H^{2}\left(g_{1}\right)$. We obtain:

$$
\begin{aligned}
n \rho_{l, i j}^{k} & =\delta_{l}^{k} \tau_{r i, j}^{r}-\delta_{l}^{k} \tau_{r j, i}^{r}+\delta_{i}^{k} \tau_{r l, j}^{r}-\delta_{j}^{k} \tau_{r l, i}^{r}-\omega^{k s}\left(\omega_{l i} \tau_{r s, j}^{r}-\omega_{l j} \tau_{r s, i}^{r}\right) \\
& =\left(\delta_{i}^{k} \tau_{l j}-\delta_{j}^{k} \tau_{l i}\right)-\omega^{k s}\left(\omega_{l i} \tau_{s j}-\omega_{l j} \tau_{s i}\right)
\end{aligned}
$$

Contracting in $k$ and $i$ while setting simply $\operatorname{tr}(\tau)=\omega^{i j} \tau_{i j}, \operatorname{tr}(\rho)=\omega^{i j} \rho_{i j}$, we get:

$$
\begin{aligned}
& n \rho_{i j}=n \tau_{i j}-\tau_{i j}-\tau_{i j}+\omega_{i j} \operatorname{tr}(\tau)=(n-2) \tau_{i j}+\omega_{i j} \operatorname{tr}(\tau)=n \rho_{j i} \\
& \Rightarrow n \operatorname{tr}(\rho)=2(n-1) \operatorname{tr}(\tau)
\end{aligned}
$$

Substituting, we finally obtain $\tau_{i j}=\frac{n}{n-2} \rho_{i j}-\frac{n}{2(n-1)(n-2)} \omega_{i j} t r(\rho)$ and thus the tricky formula:

$$
\begin{aligned}
\rho_{l, i j}^{k}= & \frac{1}{n-2}\left(\delta_{i}^{k} \rho_{l j}-\delta_{j}^{k} \rho_{l i}-\omega^{k s}\left(\omega_{l i} \rho_{s j}-\omega_{l j} \rho_{s i}\right)\right) \\
& -\frac{1}{(n-1)(n-2)}\left(\delta_{i}^{k} \omega_{l j}-\delta_{j}^{k} \omega_{l i}\right) \operatorname{tr}(\rho)
\end{aligned}
$$

Contracting in $k$ and $i$, we check that $\rho_{i j}=\rho_{i j}$ indeed, obtaining therefore the desired canonical lift $H^{2}\left(g_{1}\right) \rightarrow S_{2} T^{*} \rightarrow 0: \rho_{i, l j}^{k} \rightarrow \rho_{i, r j}^{r}=\rho_{i j}$. Finally, using Proposition 4.3, the epimorphism $H^{2}\left(g_{1}\right) \rightarrow H^{2}\left(\hat{g}_{1}\right) \rightarrow 0$ is just described by the formula:

$$
\begin{aligned}
\sigma_{l, i j}^{k}= & \rho_{l, i j}^{k}-\frac{1}{n-2}\left(\delta_{i}^{k} \rho_{l j}-\delta_{j}^{k} \rho_{l i}-\omega^{k s}\left(\omega_{l i} \rho_{s j}-\omega_{l j} \rho_{s i}\right)\right) \\
& +\frac{1}{(n-1)(n-2)}\left(\delta_{i}^{k} \omega_{l j}-\delta_{j}^{k} \omega_{l i}\right) \operatorname{tr}(\rho)
\end{aligned}
$$


which is just the way to define the Weyl tensor. We notice that $\sigma_{r, i j}^{r}=\rho_{r, i j}^{r}=0$ and $\sigma_{i, r j}^{r}=0$ by using indices or a circular chase showing that $Z^{2}\left(\hat{g}_{1}\right)=$ $Z^{2}\left(g_{1}\right)+\delta\left(T^{*} \otimes \hat{g}_{2}\right)$. This purely algebraic result only depends on the metric $\omega$ and does not depend on any conformal factor. In actual practice, the lift $H^{2}\left(g_{1}\right) \rightarrow S_{2} T^{*}$ is described by $\rho_{l, i j}^{k} \rightarrow \rho_{i, r j}^{r}=\rho_{i j}=\rho_{j i}$ but it is not evident at all that the lift $H^{2}\left(\hat{g}_{1}\right) \rightarrow H^{2}\left(g_{1}\right)$ is described by the strict inclusion $\sigma_{l, j j}^{k} \rightarrow \rho_{l, j}^{k}=\sigma_{l, j j}^{k}$ providing a short exact sequence as in Proposition 4.3 because $\rho_{i j}=\rho_{i, r j}^{r}=\sigma_{i, r j}^{r}=0$ by composition.

Q.E.D.

COROLLARY 5.4: When $n \geq 4$, each component of the Weyl tensor is a torsion element killed by the Dalembert operator $\square$ whenever the Einstein equations in vacuum are satisfied by the metric. Hence, there exists a second order operator $\mathcal{Q}$ such that we have an identity:

$$
\square \circ \text { Weyl }=\mathcal{Q} \circ \text { Ricci }
$$

Proof According to Proposition 4.4, each extension module $\operatorname{ext}^{i}(M)$ is a torsion module, $\forall i \geq 1$. It follows that each additional CC in $\mathcal{D}_{1}^{\prime}$ which is not already in $\mathcal{D}_{1}$ is a torsion element as it belongs to this module. One may also notice that:

$$
\begin{aligned}
& r k_{D}(\text { Einstein })=\frac{n(n+1)}{2}-n=\frac{n(n-1)}{2}, \\
& r k_{D}(\text { Riemann })=\frac{n(n+1)}{2}-n=\frac{n(n-1)}{2}
\end{aligned}
$$

The differential ranks of the Einstein and Riemann operators are thus equal, but this is a pure coincidence because $r k_{D}$ (Einstein) has only to do with the div operator induced by contracting the Bianchi identities, while $r k_{D}$ (Riemann) has only to do with the classical Killing operator and the fact that the corresponding differential module is a torsion module because we have a Lie group of transformations having $n+\frac{n(n-1)}{2}=\frac{n(n+1)}{2}$ parameters (translations + rotations). Hence, as the Riemann operator is a direct sum of the Weyl operator and the Einstein or Ricci operator according to the previous theorem, each component of the Weyl operator must be killed by a certain operator whenever the Einstein or Ricci equations in vacuum are satisfied. A direct tricky computation can be found in ([53], p. 206) and ([54], exercise 7.7).

Q.E.D.

REMARK 5.5: In a similar manner, the EM wave equations $\square F=0$ are easily obtained when the second set of Maxwell equations in vacuum is satisfied, avoiding therefore the Lorenz (no " $t$ ") gauge condition for the EM potential [55]. Indeed, let us start with the Minkowski constitutive law with electric constant $\epsilon_{0}$ and magnetic constant $\mu_{0}$ such that $\epsilon_{0} \mu_{0} c^{2}=1$ in vacuum:

$$
\mathcal{F}^{r s}=\frac{1}{\mu_{0}} \hat{\omega}^{r i} \hat{\omega}^{s j} F_{i j} \sim \omega^{r i} \omega^{s j} F_{i j}
$$

where $\hat{\omega}_{i j}=|\operatorname{det}(\omega)|^{-1 / n} \omega_{i j} \Rightarrow|\operatorname{det}(\hat{\omega})|=1, \quad F \in \wedge^{2} T^{*}$ is the EM field and the 
induction $\mathcal{F}$ is thus a contravariant skewsymmetric 2-tensor density. From the Maxwell equations we have:

$$
\begin{gathered}
\partial_{r} F_{i j}+\partial_{i} F_{j r}+\partial_{j} F_{r i}=0, \nabla^{r} \mathcal{F}_{r i}=0 \Rightarrow \nabla^{r} F_{r i}=0 \\
\Rightarrow \square F_{i j}=\nabla^{r} \nabla_{r} F_{i j}=\nabla^{r}\left(\nabla_{i} F_{r j}-\nabla_{j} F_{r i}\right)=0
\end{gathered}
$$

REMARK 5.6: Using Proposition 4.3 and the splittings of Theorem 5.3 for the second column, we obtain the following commutative and exact diagram:

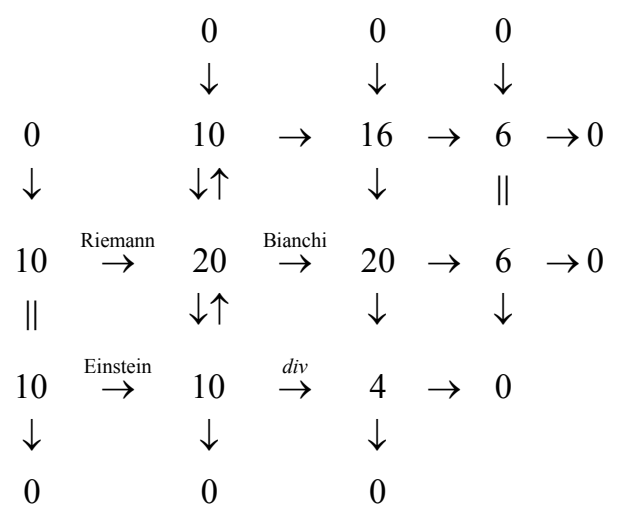

It follows that the 10 components of the Weyl tensor must satisfy a first order linear system with 16 equations, having 6 generating first order CC. The differential rank of the corresponding operator is thus equal to $16-6=10$ and such an operator defines a torsion module in which we have to look separately for each component of the Weyl tensor in order to obtain Corollary 5.4. The situation is similar to that of the Cauchy-Riemann equations when $n=2$. Indeed, any complex transformation $y=f(x)$ must be solution of the (linear) first order system $y_{2}^{2}-y_{1}^{1}=0, y_{2}^{1}+y_{1}^{2}=0$ of finite Lie equations though we obtain $y_{11}^{1}+y_{22}^{1}=0, y_{11}^{2}+y_{22}^{2}=0$, that is $y^{1}$ and $y^{2}$ are separately killed by the second order Laplace operator $\Delta=d_{11}+d_{22}$.

Collecting the above results, we obtain the striking theorem:

THEOREM 5.7: The Cauchy operator can be parametrized by the operator ad (Ricci) (with only 4 terms) and there is thus no need to introduce the Einstein operator (with 6 terms) in GR.

Proof Linearizing the Ricci tensor over the Minkowski metric, we obtain the Riccioperator $\Omega \rightarrow R$ :

$$
\begin{gathered}
2 R_{i j}=\omega^{r s}\left(d_{i j} \Omega_{r s}+d_{r s} \Omega_{i j}-d_{r i} \Omega_{s j}-d_{s j} \Omega_{r i}\right)=2 R_{j i} \\
\operatorname{tr}(R)=\omega^{i j} R_{i j}=\omega^{i j} d_{i j} \operatorname{tr}(\Omega)-\omega^{r u} \omega^{s v} d_{r s} \Omega_{u v}
\end{gathered}
$$

The Einstein operator $\Omega \rightarrow E$ is defined by setting $E_{i j}=R_{i j}-\frac{1}{2} \omega_{i j} \operatorname{tr}(R)$ that we shall write Einstein $=C \circ$ Ricci where $C: S_{2} T^{*} \rightarrow S_{2} T^{*}$ is a symmetric matrix only depending on $\omega$, which is invertible whenever $n \geq 3$. We may also introduce the linear transformation $C: \Omega \rightarrow \bar{\Omega}=\Omega-\frac{1}{2} \omega \operatorname{tr}(\Omega)$ and the unknown composite operator $\mathcal{X}: \bar{\Omega} \rightarrow \Omega \rightarrow E$ in such a way that Einstein $=\mathcal{X} \circ C$ where $\mathcal{X}$ is defined by (See [12], 5.1.5 p. 134):

$$
2 E_{i j}=\square \bar{\Omega}_{i j}-\omega^{r s} d_{r i} \bar{\Omega}_{s j}-\omega^{r s} d_{s j} \bar{\Omega}_{r i}+\omega_{i j} \omega^{r u} \omega^{s v} d_{r s} \bar{\Omega}_{u v}
$$


Now, introducing the test functions $\lambda^{i j}$, we get:

$$
\lambda^{i j} E_{i j}=\lambda^{i j}\left(R_{i j}-\frac{1}{2} \omega_{i j} t r(R)\right)=\left(\lambda^{i j}-\frac{1}{2} \omega^{i j} \omega_{r s} \lambda^{r s}\right) R_{i j}=\bar{\lambda}^{i j} R_{i j}
$$

Integrating by parts, we obtain:

$$
\left(\square \bar{\lambda}^{r s}+\omega^{r s} d_{i j} \bar{\lambda}^{i j}-\omega^{s j} d_{i j} \bar{\lambda}^{r i}-\omega^{r i} d_{i j} \bar{\lambda}^{s j}\right) \Omega_{r s}=\sigma^{r s} \Omega_{r s}
$$

Moreover, suppressing the "bar" for simplicity, we have:

$$
d_{r} \sigma^{r s}=\omega^{i j} d_{r i j} \lambda^{r s}+\omega^{r s} d_{r i j} \lambda^{i j}-\omega^{s j} d_{r i j} \lambda^{r i}-\omega^{r i} d_{r i j} \lambda^{s j}=0
$$

As Einstein is a self-adjoint operator (contrary to the Ricci operator), we have the identities:

$$
\begin{aligned}
& \operatorname{ad}(\text { Einstein })=\operatorname{ad}(C) \circ \operatorname{ad}(\mathcal{X}) \Rightarrow \text { Einstein }=C \circ \operatorname{ad}(\mathcal{X}) \\
& \Rightarrow a d(\mathcal{X})=\operatorname{Ricci} \Rightarrow \mathcal{X}=a d(\text { Ricci })
\end{aligned}
$$

because $C$ is a symmetric matrix, we have $a d(C)=C$ and we know that $a d($ Einstein $)=$ Einstein . Accordingly, the operator ad (Ricci) parametrizes the Cauchy equations, without any reference to the Einstein operator which has no mathematical origin, in the sense that it cannot be obtained by any diagram chasing. The three terms after the Dalembert operator factorize through the divergence operator $d_{i} \lambda^{r i}$. We may thus add the differential constraints $d_{i} \lambda^{r i}$ $=0$ without any reference to a gauge transformation in order to obtain a (minimum) relative parametrization (see [31] and [56] for details and explicit examples). When $n=4$ we finally obtain the adjoint sequences:

$$
\begin{aligned}
4 \stackrel{\text { Killing }}{\rightarrow} 10 & \stackrel{\text { Ricci }}{\rightarrow} 10 \\
0 \leftarrow 4 & \stackrel{\text { Cauchy }}{\leftarrow} 10 \stackrel{\operatorname{ad} \text { (Ricci) }}{\leftarrow} 10
\end{aligned}
$$

without any reference to the Bianchi operator or the induced div operator.

Q.E.D.

This last result even strengthens the doubts we already had about the origin and existence of gravitational waves.

\section{Conclusions}

Whenever $R_{q} \subseteq J_{q}(E)$ is an involutive system of order $q$ on $E$, we may define the Janet bundles $F_{r}$ for $r=0,1, \cdots, n$ by the short exact sequences:

$$
0 \rightarrow \wedge^{r} T^{*} \otimes R_{q}+\delta\left(\wedge^{r-1} T^{*} \otimes S_{q+1} T^{*} \otimes E\right) \rightarrow \wedge^{r} T^{*} \otimes J_{q}(E) \rightarrow F_{r} \rightarrow 0
$$

We may pick up a section of $F_{r}$, lift it up to a section of $\wedge^{r} T^{*} \otimes J_{q}(E)$ that we may lift up to a section of $\wedge^{r} T^{*} \otimes J_{q+1}(E)$ and apply $D$ in order to get a section of $\wedge^{r+1} T^{*} \otimes J_{q}(E)$ that we may project onto a section of $F_{r+1}$ in order to construct an operator $\mathcal{D}_{r+1}: F_{r} \rightarrow F_{r+1}$ generating the $\mathrm{CC}$ of $\mathcal{D}_{r}$ in the canonical linear Janet sequence ([5], p. 145):

$$
0 \rightarrow \Theta \rightarrow E \stackrel{\mathcal{D}}{\rightarrow} F_{0} \stackrel{\mathcal{D}_{1}}{\rightarrow} F_{1} \stackrel{\mathcal{D}_{2}}{\rightarrow} \cdots \stackrel{\mathcal{D}_{n}}{\rightarrow} F_{n} \rightarrow 0
$$


If we have two involutive systems $R_{q} \subset \hat{R}_{q} \subset J_{q}(E)$, the Janet sequence for $R_{q}$ projects onto the Janet sequence for $\hat{R}_{q}$ and we may define inductively canonical epimorphisms $F_{r} \rightarrow \hat{F}_{r} \rightarrow 0$ for $r=0,1, \cdots, n$ by comparing the previous sequences for $R_{q}$ and $\hat{R}_{q}$.

A similar procedure can also be obtained if we define the Spencer bundles $C_{r}$ for $r=0,1, \cdots, n$ by the short exact sequences:

$$
0 \rightarrow \delta\left(\wedge^{r-1} T^{*} \otimes g_{q+1}\right) \rightarrow \wedge^{r} T^{*} \otimes R_{q} \rightarrow C_{r} \rightarrow 0
$$

We may pick up a section of $C_{r}$, lift it to a section of $\wedge^{r} T^{*} \otimes R_{q}$, lift it up to a section of $\wedge^{r} T^{*} \otimes R_{q+1}$ and apply $D$ in order to construct a section of $\wedge^{r+1} \otimes R_{q}$ that we may project to $C_{r+1}$ in order to construct an operator $D_{r+1}: C_{r} \rightarrow C_{r+1}$ generating the CC of $D_{r}$ in the canonical linear Spencer sequence which is another completely different resolution of the set $\Theta$ of (formal) solutions of $R_{q}$ :

$$
0 \rightarrow \Theta \stackrel{j_{q}}{\rightarrow} C_{0} \stackrel{D_{1}}{\rightarrow} C_{1} \stackrel{D_{2}}{\rightarrow} C_{2} \stackrel{D_{3}}{\rightarrow} \cdots \stackrel{D_{n}}{\rightarrow} C_{n} \rightarrow 0
$$

However, if we have two systems as above, the Spencer sequence for $R_{q}$ is now contained into the Spencer sequence for $\hat{R}_{q}$ and we may construct inductively canonical monomorphisms $0 \rightarrow C_{r} \rightarrow \hat{C}_{r}$ for $r=0,1, \cdots, n$ by comparing the previous sequences for $R_{q}$ and $\hat{R}_{q}$.

When dealing with applications, we have set $E=T$ and considered systems of finite type Lie equations determined by Lie groups of transformations and $\operatorname{ad}\left(\mathcal{D}_{r}\right)$ generates the CC of $\operatorname{ad}\left(\mathcal{D}_{r+1}\right)$ while $\operatorname{ad}\left(D_{r}\right)$ generates the CC of $\operatorname{ad}\left(D_{r+1}\right)$. We have obtained in particular $C_{r}=\wedge^{r} T^{*} \otimes R_{q} \subset \wedge^{r} T^{*} \otimes \hat{R}_{q}=\hat{C}_{r}$ when comparing the classical and conformal Killing systems, but these bundles have never been used in physics. Therefore, instead of the classical Killing system $R_{2} \subset J_{2}(T)$ defined by $\Omega \equiv \mathcal{L}(\xi) \omega=0$ and $\Gamma \equiv \mathcal{L}(\xi) \gamma=0$ or the conformal Killing system $\hat{R}_{2} \subset J_{2}(T)$ defined by $\Omega \equiv \mathcal{L}(\xi) \omega=A(x) \omega$ and $\Gamma \equiv \mathcal{L}(\xi) \gamma=\left(\delta_{i}^{k} A_{j}(x)+\delta_{j}^{k} A_{i}(x)-\omega_{i j} \omega^{k s} A_{s}(x)\right) \in S_{2} T^{*} \otimes T$, we may introduce the intermediate differential system $\tilde{R}_{2} \subset J_{2}(T)$ defined by $\mathcal{L}(\xi) \omega=A \omega$ with $A=c s t$ and $\Gamma \equiv \mathcal{L}(\xi) \gamma=0$, for the Weyl group obtained by adding the only dilatation with infinitesimal generator $x^{i} \partial_{i}$ to the Poincare group. We have $R_{1} \subset \tilde{R}_{1}=\hat{R}_{1}$ but the strict inclusions $R_{2} \subset \tilde{R}_{2} \subset \hat{R}_{2}$ and we discover exactly the group scheme used through this paper, both with the need to shift by one step to the left the physical interpretation of the various differential sequences used. Indeed, as $\hat{g}_{2} \simeq T^{*}$, the first Spencer operator $\hat{R}_{2} \stackrel{D_{1}}{\rightarrow} T^{*} \otimes \hat{R}_{2}$ is induced by the usual Spencer operator $\hat{R}_{3} \stackrel{D}{\rightarrow} T^{*} \otimes \hat{R}_{2}:\left(0,0, \xi_{r j}^{r}, \xi_{r i j}^{r}=0\right) \rightarrow$ $\left(0, \partial_{i} 0-\xi_{r i}^{r}, \partial_{i} \xi_{r j}^{r}-0\right)$ and thus projects by cokernel onto the induced operator $T^{*} \rightarrow T^{*} \otimes T^{*}$. Composing with $\delta$, it projects therefore onto $T^{*} \stackrel{d}{\rightarrow} \wedge^{2} T^{*}: A \rightarrow$ $d A=F$ as in EM and so on by using the fact that $D_{1}$ and $d$ are both involutive or the composite epimorphisms

$\hat{C}_{r} \rightarrow \hat{C}_{r} / \tilde{C}_{r}=\wedge^{r} T^{*} \otimes\left(\hat{R}_{2} / \tilde{R}_{2}\right) \simeq \wedge^{r} T^{*} \otimes \hat{g}_{2} \simeq \wedge^{r} T^{*} \otimes T^{*} \stackrel{\delta}{\rightarrow} \wedge^{r+1} T^{*}$. The main result we have obtained is thus to be able to increase the order and dimension of the underlying jet bundles and groups, proving therefore that any 1-form with 
value in the second order jets $\hat{g}_{2}$ (elations) of the conformal Killing system (conformal group) can be decomposed uniquely into the direct sum $(R, F)$ where $R$ is a section of the Ricci bundle $S_{2} T^{*}$ and the EM field $F$ is a section of $\wedge^{2} T^{*}$ as conjectured by H. Weyl in 1918 [13] [14] [57].

The mathematical structures of electromagnetism and gravitation only depend on the second order jets.

\section{References}

[1] Pommaret, J.-F. (1995) Comptes Rendus Acadéemie des Sciences Paris, 320, 1225-1230.

[2] Pommaret, J.-F. (1978) Systems of Partial Differential Equations and Lie Pseudogroups. Gordon and Breach, New York; Russian Translation, MIR, Moscow.

[3] Pommaret, J.-F. (1983) Differential Galois Theory. Gordon and Breach, New York.

[4] Pommaret, J.-F. (1988) Lie Pseudogroups and Mechanics. Gordon and Breach, New York.

[5] Pommaret, J.-F. (1994) Partial Differential Equations and Group Theory. Kluwer, Dordrecht. https://doi.org/10.1007/978-94-017-2539-2

[6] Pommaret, J.-F. (2016) Deformation Theory of Algebraic and Geometric Structures. Lambert Academic Publisher (LAP), Saarbrucken, Germany. http://arxiv.org/abs/1207.1964

[7] Vessiot, E. (1903) Annales Ecole Normale Supéerieure, 20, 411-451. http://numdam.org

[8] Airy, G.B. (1863) Philosophical Transactions of the Royal Society, London, 153, 49-80. https://doi.org/10.1098/rstl.1863.0004

[9] Duc, J. and Bellet, D. (1976) Mécanique des solides réels-Elasticité, SUP'AERO Course. Cepadues Editions, Toulouse.

[10] Duc, J. and Bellet, D. (1976) Problèmes d'Elasticité, SUP'AERO Course. Cepadues Editions, Toulouse.

[11] Pommaret, J.-F. (2001) Partial Differential Control Theory. Kluwer, Dordrecht (1000 pp). https://doi.org/10.1007/978-94-010-0854-9

[12] Foster, J. and Nightingale, J.D. (1979) A Short Course in General Relativity, Longman, New York.

[13] Pommaret, J.-F. (2014) Journal of Modern Physics, 5, 157-170. https://doi.org/10.4236/jmp.2014.55026

[14] Pommaret, J.-F. (2015) From Thermodynamics to Gauge Theory: The Virial Theorem Revisited. In: Gauge Theories and Differential Geometry, NOVA Science Publisher, 1-46.

[15] Malliavin, M.-P. (1989-1990) Topics in Invariant Theory. Springer, Berlin, 244-254.

[16] Kashiwara, M. (1995) Algebraic Study of Systems of Partial Differential Equations, Mémoires de la Société Mathématique de France, 63 (Transl. from Japanese of His 1970 Masters Thesis).

[17] Assem, I. (1997) Algèbres et Modules. Masson, Paris.

[18] Bourbaki, N. (1980) Algèbre, Ch. 10, Algèbre Homologique. Masson, Paris.

[19] Hu, S.-T. (1968) Introduction to Homological Algebra. Holden-Day, San Francisco. 
[20] Northcott, D.G. (1966) An Introduction to Homological Algebra. Cambridge University Press, Cambridge.

[21] Rotman, J.J. (1979) An Introduction to Homological Algebra, Pure and Applied Mathematics. Academic Press (Springer for Second Edition), New York.

[22] Oberst, U. (1990) Acta Applicandae Mathematicae, 20, 1-175. https://doi.org/10.1007/BF00046908

[23] Oberst, U. (2013) Multidim Syst Sign Process (MSSP), 26, 89-404.

[24] Pommaret, J.-F. (2005) Algebraic Analysis of Control Systems Defined by Partial Differential Equations. In: Advanced Topics in Control Systems Theory, Lecture Notes in Control and Information Sciences 311, Chapter 5, Springer, 155-223. https://doi.org/10.1007/11334774_5

[25] Zerz, E. (2000) Topics in Multidimensional Linear Systems Theory. Lecture Notes in Control and Information Sciences (LNCIS) 256, Springer.

[26] Pommaret, J.-F. and Quadrat, A. (1999) Systems \& Control Letters, 37, 247-260. https://doi.org/10.1016/S0167-6911(99)00030-4

[27] Pommaret, J.-F. and Quadrat, A. (1999) IMA Journal of Mathematical Control and Information, 16, 275-297. https://doi.org/10.1093/imamci/16.3.275

[28] Maxwell, J.C. (1870) Transactions of the Royal Society of Edinburgh, 26, 1-40. https://doi.org/10.1017/S0080456800026351

[29] Morera, G. (1892) Atti della Reale Accademia dei Lincei, 1, 137-141, 233.

[30] Beltrami, E. (1892) Atti della Reale Academia dei Lincei, 5, 141-142.

[31] Pommaret, J.-F. (2016) Journal of Modern Physics, 7, 699-728. https://doi.org/10.4236/jmp.2016.77068

[32] Chyzak, F., Quadrat, A. and Robertz, D. (2016) Applicable Algebra in Engineering, Communication and Computing, 16, 319-376. https://doi.org/10.1007/s00200-005-0188-6

[33] Chyzak, F., Quadrat, A. and Robertz, D. (2007) Lecture Notes in Control and Information Sciences, 352, 233-264. http://wwwb.math.rwth-aachen.de/OreModules

[34] Quadrat, A. (2010) Les cours du CIRM, Journees Nationales de Calcul Formel, 1, 281-471.

[35] Quadrat, A. and Robertz, R. (2014) Acta Applicandae Mathematicae, 133, 187-234. http://hal-supelec.archives-ouvertes.fr/hal-00925533

[36] Eisenhart, L.P. (1926) Riemannian Geometry. Princeton University Press, Princeton.

[37] Goldschmidt, H. (1969) Journal of Differential Geometry, 1, 269-307. https://doi.org/10.4310/jdg/1214428094

[38] Janet, M. (1920) Journal de Mathématiques Pures et Appliquées, 3, 65.

[39] Spencer, D.C. (1965) Bulletin of the AMS, 75, 1-114.

[40] Kaplansky, I. (1957) An Introduction to Differential Algebra. Hermann, Paris.

[41] Kolchin, E.R. (1973) Differential Algebra and Algebraic Groups. Academic Press, New York.

[42] Ritt, J.F. (1960) Differential Algebra. Dover, New York.

[43] Bialynicki-Birula, A. (1961) Pacific Journal of Mathematics, 11, 1205-1209. https://doi.org/10.2140/pjm.1961.11.1205

[44] Bialynicki-Birula, A. (1962) American Journal of Mathematics, 84, 89-109. 
https://doi.org/10.2307/2372805

[45] Vessiot, E. (1904) Annales Ecole Normale Supéerieure, 21, 9-85. http://numdam.org

[46] Bjork, J.E. (1993) Analytic D-Modules and Applications. Kluwer, Dordrecht. https://doi.org/10.1007/978-94-017-0717-6

[47] Schneiders, J.-P. (1994) Bulletin de la Société Royale des Sciences de Liège, 63, 223-295.

[48] Kunz, E. (1985) Introduction to Commutative Algebra and Algebraic Geometry. Birkhaser, Boston.

[49] Cosserat, E. and Cosserat, F. (1909) Théorie des Corps Déformables. Hermann, Paris.

[50] Pommaret, J.-F. (2010) Acta Mechanica, 215, 43-55. https://doi.org/10.1007/s00707-010-0292-y

[51] Pommaret, J.-F. (2012) Spencer Operator and Applications: From Continuum Mechanics to Mathematical Physics. In: Gan, Y., Ed., Continuum Mechanics-Progress in Fundamentals and Engineering Applications, Intech, Rijeka (Croatia) 1-32. https://doi.org/10.5772/35607

[52] Pommaret, J.-F. (2013) Journal of Modern Physics, 4, 223-239. https://doi.org/10.4236/jmp.2013.48A022

[53] Choquet-Bruhat, Y. (2015) Introduction to General Relativity, Black Holes and Cosmology. Oxford University Press, Oxford.

[54] Hughston, L.P. and Tod, K.P. (1990) An Introduction to General Relativity, London Math. Soc. Students Texts 5. Cambridge University Press, Cambridge.

[55] Ougarov, V. (1969) Théorie de la Relativité Restreinte. MIR, Moscow (French Translation, 1979).

[56] Pommaret, J.-F. (2015) Multidimensional Systems and Signal Processing, 26, 405-437. https://doi.org/10.1007/s11045-013-0265-0

[57] Weyl, H. (1918) Space, Time, Matter. Springer, 1958; Dover, 1952. 